DOI: $10.24850 / j-$ tyca-2020-05-06

Artículos

\title{
Efectos del otorgamiento de derechos de agua en la disponibilidad de recursos hídricos en la cuenca del río Ñuble, Chile Centro Sur
}

Effects of water rights allocation on water resources availability within the Nuble River Basin, South Central Chile

Iván Belmar ${ }^{1}$

Alfonso Fernández², ORCID: https://orcid.org/0000-0001-6825-0426 Gianni Leal ${ }^{3}$

${ }^{1}$ Departamento de Geografía, Universidad de Concepción, Concepción, Chile, ivanbelmarvejar@gmail.com

2Departamento de Geografía \& Mountain Geoscience Group, Universidad de Concepción, Concepción, Chile, alfernandez@udec.cl

${ }^{3}$ Departamento de Geografía \& Mountain Geoscience Group, Universidad de Concepción, Concepción, Chile, glealparra@gmail.com

Autor para correspondencia: Alfonso Fernández, alfernandez@udec.cl 


\section{Resumen}

Se analiza la disponibilidad de agua para la constitución de derechos de aprovechamiento de la misma en la cuenca del río Ñuble, Chile, utilizando una metodología que evalúa el recurso en régimen natural, determina la demanda existente y genera un balance hídrico. Para el cálculo de la oferta hídrica se utilizaron registros de precipitación de la misión TRMM (por sus siglas en inglés, Misión de Medición de Precipitaciones Tropicales), a lo que se suman registros instrumentales de estaciones fluviométricas y pluviométricas. Para la demanda se realizó un análisis de la totalidad de los derechos concedidos en el área de estudio. Los resultados revelan la existencia de déficit hídrico. Específicamente, existe un sobreotorgamiento de derechos de agua entre los meses de octubre y mayo, con déficits de hasta $7.8 \mathrm{~m}^{3} / \mathrm{s}$ para derechos de ejercicio permanente y $63.7 \mathrm{~m}^{3} / \mathrm{s}$, sumando los derechos de ejercicio eventual. Una mejora en el otorgamiento de derechos debiera contemplar estimaciones más confiables de balance hídrico, incorporando registros actualizados y validados de derechos de agua, y más estaciones hidrometeorológicas en cuencas de gran altitud. Además, se propone que es necesario que el Estado cuente con mayores atribuciones para una gestión eficiente del agua, en un escenario hidroclimático con sequías cada vez más recurrentes.

Palabras clave: derechos de agua, disponibilidad de agua, balance hídrico.

\section{Abstract}


In this study, we analyze water availability for allocation of water rights in Ñuble River Basin, Chile. The methods include evaluation of the natural regime, determination of current water demand, and calculation of a water budget. Water availability was calculated using TRMM and instrumental records from rainfall and discharge gauges. Water demand was computed from a new inventory of water rights records developed for this study. Results reveal a hydrological deficit. More specifically, May and October showed the highest deficit, with $7.8 \mathrm{~m} 3 / \mathrm{s}$ for permanent water rights and $63.7 \mathrm{~m} 3 / \mathrm{s}$ for sporadic water rights. In order to reduce this deficit the method to allocate water right should include better estimations of hydrological balances by incorporating updated and validated water rights records and more hydrometeorological instrumentation in mountain watersheds. Furthermore, we assert that the government must play a bigger role in efficient water management, considering the present scenario of more frequent droughts.

Keywords: Water rights, water availability, hydrological balance.

Recibido: 02/09/2019

Aceptado: $18 / 02 / 2020$

\section{Introducción}


Desde el año 2010, una extensa región de Chile central ( $\sim 30^{\circ} \mathrm{S}$ a $39^{\circ}$ S) ha sufrido uno de sus periodos más secos y cálidos de los últimos cien años (CR2, 2015), con un déficit hídrico que ha impactado fuertemente actividades agrícolas, energéticas y de disponibilidad de agua para consumo humano (Garreaud et al., 2017). Aunque se sabe que eventos de sequía han afectado esta región con regularidad (Muñoz et al., 2016), existe una alta probabilidad de que una parte significativa de eventos de déficit sean consecuencia del cambio climático antrópico (Boisier, Rondanelli, Garreaud, \& Muñoz, 2016). Ello sugiere que en el futuro estos eventos se pueden constituir en el régimen hídrico normal, exacerbando el déficit hídrico a través de la evaporación desde lagos, embalses y cultivos.

Lo expuesto en el párrafo anterior se relaciona directamente con el régimen hídrico natural, en que la precipitación (líquida y sólida) alimenta ríos y acuíferos y, por lo tanto, determina la oferta hídrica. Otro componente de esta problemática es la demanda hídrica. En Chile, el acceso al agua superficial se rige por el Código de Aguas de 1981. Este marco legal, encargado de normar los usos de los recursos hídricos, ha operado como fuente de derecho privado sobre el territorio, reforzando el uso productivo del recurso (Bolados, Henríquez, Ceruti, \& Sánchez, 2017) y se guía por la teoría del libre mercado, asignando un rol subsidiario al Estado (Ugarte, 2003). El elemento que determina la facultad de disponer del recurso es el derecho de aprovechamiento de 
agua (en adelante DdA), otorgado de forma gratuita, a perpetuidad; se puede separar del derecho de propiedad a la tierra (Budds, 2012; Bolados et al., 2017) y ser transferido a través del mercado (Vergara, 2015; Costa, 2016). En este contexto, es relevante el concepto de DdA consuntivo, que según el artículo $13^{\circ}$ del Código de Aguas de Chile (Ministerio de Justicia, 2018): "Son aquellos que facultan a su titular para consumir totalmente las aguas".

En la actualidad, se discute una reforma a este código, donde se destaca la asignación de uso prioritario del agua para el consumo humano, la protección de áreas de importancia ambiental y el fortalecimiento de las atribuciones de la administración. Sin embargo, la propuesta de reforma tiene puntos críticos que están en discusión, como la afectación de la norma sobre los DdA vigentes, los plazos de concesiones, las extinciones de DdA, y la dificultad en la aplicación de caudales ecológicos (González, 2017).

Por otra parte, Vergara (2015) reconoce una notable debilidad del actual sistema chileno, donde todavía existe una gran proporción de DdA reconocidos, pero que no están inscritos ni regularizados en el registro de catastro público alguno. Lo anterior indica que el método de asignación del recurso es ineficiente debido a la incertidumbre en los datos (Bolados et al., 2017), lo que puede generar errores en el otorgamiento de nuevos $\mathrm{DdA}$, y repercutir de manera directa en la disponibilidad hídrica.

Una mejora en las bases de datos de DdA se traduciría en análisis de disponibilidad más precisos. Esto se hace indispensable en un 
contexto de reforma, pues se busca que el estado resguarde la existencia de un caudal suficiente en las fuentes naturales, velando por sus funciones escénicas, paisajísticas, ambientales y sociales (González, 2017).

La presente investigación busca evaluar, con base en la información existente en fuentes de entidades públicas de Chile, la disponibilidad para la asignación de nuevos DdA consuntivos en la cuenca del río Ñuble, en Chile Centro Sur ( $\left.36^{\circ} \mathrm{S}\right)$. El análisis de los DdA indica que en algunos de ellos no están los registros de los expedientes de su constitución y, por tanto, no se tiene certeza de su existencia real (ver la sección de materiales y métodos). Este trabajo desarrolló una metodología que permitió incluir esos posibles DdA; de esta forma, se utilizaron dos escenarios de caudales de DdA: uno en que sólo se consideraron los DdA que contienen información verificada de caudales, y otro en que aquellos fueron analizados en conjunto con Ios DdA que no se tiene certeza de su constitución. Estos dos escenarios permitieron evaluar la sensibilidad de la cuenca del río Nuble a una situación extrema, en que todos DdA consuntivos se utilizaran totalmente, considerando que un aumento del uso de agua frente a condiciones de escasez incremental puede generar conflictos (Valdés-Pineda et al., 2014).

\section{Materiales y métodos}


Tecnología y

Ciencias $₫$ Agua
2020, Instituto Mexicano de Tecnología del Agua

Open Access bajo la licencia CCBY-NC-SA 4.0

(https://creativecommons.org/licenses/by-nc-sa/4.0/)

\section{Área de estudio}

El área de estudio comprende la cuenca del río Ñuble, la cual posee un $38.6 \%$ del área total de la región en la que se emplaza (región de Ñuble). Sus principales actividades económicas son la agricultura y silvicultura (DGA, 2004b), y es aquí donde se sitúa la décimo segunda ciudad con más habitantes de Chile (unos 200000 habitantes), la ciudad de Chillán (MINVU, 2002). Se ubica entre las coordenadas $36.29^{\circ}$ y $37.33^{\circ}$ de latitud sur y entre los $71^{\circ}$ y $72.46^{\circ}$ de longitud oeste, en la región de Ñuble, zona centro sur de Chile (Figura 1 ).

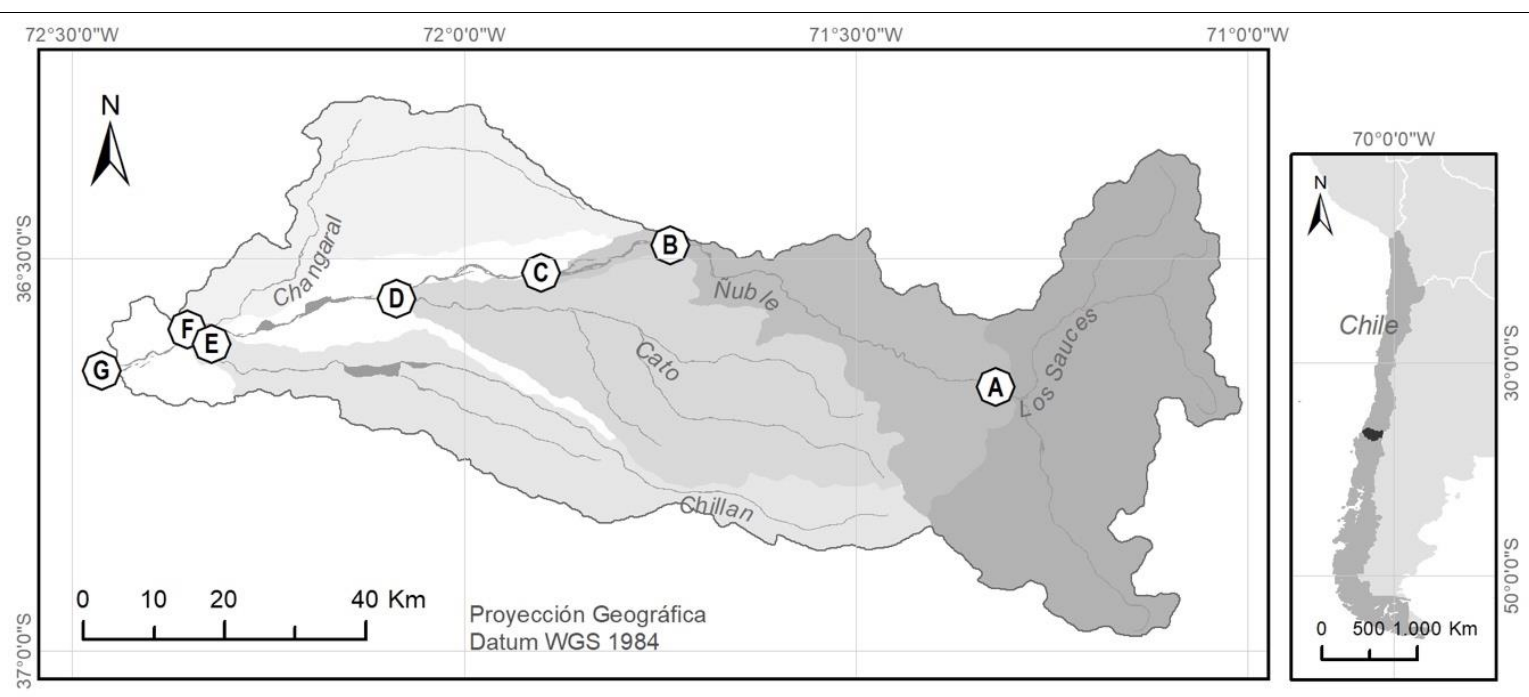


2020, Instituto Mexicano de Tecnología del Agua

Open Access bajo la licencia CC BY-NC-SA 4.0

(https://creativecommons.org/licenses/by-nc-sa/4.0/)

Figura 1. Área de estudio cuenca del río Ñuble, subcuencas y sus puntos de control. Las subcuencas se muestran como áreas con distintas tonalidades de grises y son identificadas por el nombre de su río.

El río Ñuble, con una longitud de 155 km, posee una cuenca hidrográfica de 5097 km² (DGA, 2004b).

El sector oriental del área de estudio se emplaza en la cordillera de Los Andes y el sector occidental en la depresión intermedia, la cual es una franja del relieve chileno que, por su extensión como rasgo continuo, facilita el asentamiento de gran parte de la población (Universidad de Chile, s.f.).

Esta cuenca, influenciada por un clima mediterráneo, presenta al menos dos meses consecutivos del periodo estival con déficit hídrico, cuando es más demandado el recurso hídrico. En 1991, la cuenca presentaba un total de 1119 canales, que servían para el riego de 562 $\mathrm{km}^{2}$ (DGA, 2004b), equivalente al $11 \%$ de la superficie total de ésta.

El área de estudio se dividió en siete puntos de control, en los que se determinó su disponibilidad: las desembocaduras de los ríos Cato, Chillán, Changaral y Ñuble ( $D, E, F$ y $G$ en la Figura 1), más tres puntos adicionales de interés en el río Ñuble ( $A, B$ y $C$ en la Figura 1). Los puntos situados en las desembocaduras se seleccionaron debido a que son los afluentes más importantes (DGA, 2004b), y los puntos de interés del río Ñuble se escogieron porque presentan DdA con caudales concedidos de gran magnitud en sus subcuencas aportantes. 


\section{Evaluación de disponibilidad hídrica}

Para la evaluación hídrica se debe hacer un balance entre la oferta y la demanda de agua, lo que da como resultado la disponibilidad de caudales para la constitución de nuevos DdA en los puntos evaluados (DGA, 2008). Esto es evaluado tanto para DdA de ejercicio permanente como eventual. Por una parte, el artículo $17^{\circ}$ del Código de Aguas de Chile (DFL, 2018) dice que los derechos de ejercicio permanente: "Son aquellos que facultan para usar el agua en la dotación que corresponda". Por otra parte, el artículo $18^{\circ}$ menciona a los DdA de ejercicio eventual como aquellos que: "Sólo facultan para usar el agua en las épocas en que el caudal matriz tenga un sobrante después de abastecidos los derechos de ejercicio permanente".

\section{Oferta hídrica}

Para realizar el análisis de disponibilidad es necesario estimar los recursos superficiales en régimen natural (DGA, 2004a), es decir, se 
debe considerar el agua que pasaría por el punto si no tuviera ninguna extracción o intervención humana.

Para esto, se aplicó la metodología de transposición de cuencas por área y precipitación, pues tal procedimiento es lo que recomienda el Manual de Normas y Procedimientos para la Administración de Recursos Hídricos de la Dirección General de Aguas (DGA). Sin bien dicha metodología no es nueva, es recomendada y adecuada para los fines que busca este trabajo (DGA, 2008). Es una metodología que se utiliza para determinar caudales en cuencas no instrumentadas (ÁlvarezSevilla, Brown-Manrique, \& del Cid-Colindres, 2017; Duitama, Moreno, \& Zamudio, 2015), donde otros métodos, como la correlación caudal precipitación o el balance hídrico, son imposible de aplicar por la falta de datos. En efecto, dicha metodología estima el caudal en régimen natural que pasaría por un punto evaluado utilizando registros de estaciones fluviométricas patrón, las cuales corresponden a estaciones que, por la cercanía a los puntos por evaluar, la similitud con sus regímenes y por las nulas extracciones que poseen en sus subcuencas aportantes, se seleccionaron para determinar los caudales teóricos que pasarían por los puntos evaluados. Este método asume que el punto evaluado posee el mismo régimen de distribución de caudales que el de la estación patrón, y que la relación se establece con base en el tamaño de la subcuenca que conforma y a la precipitación media anual de ésta. La Ecuación (1) que describe el cálculo es la siguiente:

$q_{j i}=\frac{Q_{i j} \times a \times p}{A \times P}$ 
2020, Instituto Mexicano de Tecnología del Agua

Open Access bajo la licencia CC BY-NC-SA 4.0

(https://creativecommons.org/licenses/by-nc-sa/4.0/)

donde qij: caudal medio mensual a estimar para un mes $i$ y una probabilidad de excedencia (en adelante PE) $j$; Qij: caudal medio mensual de la cuenca patrón para un mes $i$ (dato que proviene de registros existentes), y una $\operatorname{PE} j ; a$ : área drenada de la cuenca evaluada; $p$ : precipitación media anual de la cuenca evaluada; $A$ : área drenada de la cuenca patrón; $P$ : precipitación media anual de la cuenca patrón. Para estimar el parámetro $a$ de cada subcuenca del río Ñuble, se delimitaron los límites de cuenca del río Ñuble y sus subcuencas (Figura 1) utilizando la herramienta ArcHydro Tools del software ArcGIS 10.3 (ESRI, 2011). La base topográfica para este procedimiento fue el modelo digital de elevaciones SRTM (Shuttle Radar Topography Mission), con una resolución espacial de 1 arco segundo ( 30 metros). Este modelo se descargó de la plataforma web Earth Explorer del US Geological Survey (USGS, 2014). El producto descargado se procesó con el cálculo de precipitaciones y caudales que se detallan en los siguientes apartados.

La aplicación de la Ecuación (1) dio como resultado la oferta hídrica, es decir, los caudales medios mensuales en régimen natural a Q10PE, Q85PE y Q95PE de cada punto evaluado; esto es, caudal con probabilidad de excedencia de 10,85 y $95 \%$, respectivamente.

\section{Ajuste de precipitaciones}


Tecnología y

Ciencias $₫$ Agua
2020, Instituto Mexicano de Tecnología del Agua

Open Access bajo la licencia CC BY-NC-SA 4.0

(https://creativecommons.org/licenses/by-nc-sa/4.0/)

Para el análisis de la precipitación por cada subcuenca del área de estudio (Figura 1) es necesario la existencia de registros instrumentales. Sin embargo, según la Tabla 1, elaborada con los registros del Centro de Ciencia del Clima y la Resiliencia (CR2, 2017), el área de estudio sólo contiene 24 estaciones pluviométricas, con un mínimo de $80 \%$ de datos durante el intervalo de análisis considerado de 1991 a 2016 (ver la subsección sobre estaciones fluviométricas patrón), las cuales poseen una distribución espacial que dificulta el estudio a la escala de subcuenca.

Tabla 1. Estaciones pluviométricas.

\begin{tabular}{|c|c|c|c|c|c|c|c|}
\hline \multirow{2}{*}{ Código } & Nombre & \multicolumn{2}{|c|}{ Coordenadas } & \multicolumn{2}{c|}{$\begin{array}{c}\text { Años de } \\
\text { Observación }\end{array}$} & $\begin{array}{c}\text { Altitud } \\
\text { (msnm) }\end{array}$ & $\begin{array}{c}\text { Precipitación } \\
\text { (mm /año) }\end{array}$ \\
\cline { 3 - 7 } & & & Oeste & Sur & Inicio & Fin & \\
\hline 1 & Nueva Aldea & $72.4561^{\circ}$ & $36.6544^{\circ}$ & 1956 & 2016 & 32 & 963 \\
\hline 2 & Chillancito & $72.4239^{\circ}$ & $36.7622^{\circ}$ & 1954 & 2016 & 56 & 1013 \\
\hline 3 & Chillán Viejo & $72.1267^{\circ}$ & $36.6319^{\circ}$ & 1977 & 2017 & 115 & 941 \\
\hline 4 & Ma. Dolores & $72.4225^{\circ}$ & $37.4028^{\circ}$ & 1951 & 2017 & 120 & 1009 \\
\hline 5 & San Agustín & $72.3944^{\circ}$ & $36.4214^{\circ}$ & 1993 & 2016 & 138 & 866 \\
\hline 6 & Las Achiras & $72.3661^{\circ}$ & $37.3806^{\circ}$ & 1964 & 2016 & 144 & 933 \\
\hline 7 & B. O'Higgins & $72.04^{\circ}$ & $36.5872^{\circ}$ & 1950 & 2017 & 151 & 891 \\
\hline 8 & Millauquén & $72.04^{\circ}$ & $36.3183^{\circ}$ & 1992 & 2016 & 152 & 861 \\
\hline
\end{tabular}


Tecnología y

Ciencias $₫$ Agua
2020, Instituto Mexicano de Tecnología del Agua

Open Access bajo la licencia CC BY-NC-SA 4.0

(https://creativecommons.org/licenses/by-nc-sa/4.0/)

\begin{tabular}{|c|c|c|c|c|c|c|c|}
\hline 9 & Pemuco & $72.1008^{\circ}$ & $36.9764^{\circ}$ & 1971 & 2016 & 200 & 140 \\
\hline 10 & Cholguán & $72.0669^{\circ}$ & $37.1528^{\circ}$ & 1956 & 2016 & 230 & 1247 \\
\hline 11 & San Manuel & $71.6494^{\circ}$ & $36.3581^{\circ}$ & 1956 & 2017 & 270 & 1470 \\
\hline 12 & Coihueco & $71.7989^{\circ}$ & $36.6408^{\circ}$ & 1900 & 2016 & 314 & 1392 \\
\hline 13 & Tucapel & $71.9519^{\circ}$ & $37.2936^{\circ}$ & 1975 & 2016 & 342 & 1469 \\
\hline 14 & Mayulermo & $71.8944^{\circ}$ & $36.8189^{\circ}$ & 1992 & 2016 & 371 & 1462 \\
\hline 15 & Digua & $71.5481^{\circ}$ & $36.2558^{\circ}$ & 1947 & 2017 & 390 & 1280 \\
\hline 16 & San Fabián & $71.5247^{\circ}$ & $36.5836^{\circ}$ & 1956 & 2016 & 465 & 1512 \\
\hline 17 & Trupán & $71.8228^{\circ}$ & $37.2806^{\circ}$ & 1963 & 2016 & 474 & 1625 \\
\hline 18 & Las Cruces & $71.765^{\circ}$ & $37.1114^{\circ}$ & 1993 & 2016 & 476 & 1782 \\
\hline 19 & Bullileo & $71.4142^{\circ}$ & $36.285^{\circ}$ & 1930 & 2017 & 600 & 1985 \\
\hline 20 & Caracol & $71.395^{\circ}$ & $36.6511^{\circ}$ & 1900 & 2016 & 610 & 2287 \\
\hline 21 & Caman & $71.2989^{\circ}$ & $36.6731^{\circ}$ & 1992 & 2016 & 670 & 2131 \\
\hline 22 & Diguillín & $71.6425^{\circ}$ & $36.8686^{\circ}$ & 1900 & 2016 & 670 & 2042 \\
\hline 23 & Fdo. Atacalco & $71.5814^{\circ}$ & $36.9175^{\circ}$ & 1930 & 2016 & 740 & 2131 \\
\hline
\end{tabular}

Por esta razón, se optó por utilizar un método de regionalización de la precipitación a datos instrumentales y satelitales. La Misión de Medición de Precipitaciones Tropicales (TRMM, por sus siglas en inglés) proporciona un producto de resolución espacial de $0.25^{\circ}$ y con periodicidad mensual (NASA, 2015). Con estos datos mensuales en formato raster (grilla), obtenidos de la interfase web de la NASA, Giovanni (acrónimo del inglés para Goddard Earth Sciences Data and Information Services Center Interactive Online Visualization and 
Analysis Infrastructure), que provee información de varios satélites para todas las zonas del mundo (NASA, 2017), se calcularon totales anuales para el periodo 1998-2017. Tales valores de precipitación se compararon con los registros de las estaciones pluviométricas presentadas en la Tabla 1, a fin de determinar el sesgo y ejecutar correcciones estadísticas, las cuales se llevaron a cabo mediante el método de corrección por regresión lineal (Canavos, 1998).

Por último, se determinó la precipitación media anual de cada subcuenca de estudio mediante el promedio de la precipitación de los pixeles del producto TRMM corregido que se localicen en cada subcuenca del río Ñuble. Esta precipitación se utilizó en la Ecuación (1) de transposición de cuencas.

\section{Estaciones fluviométricas patrón}

Con base en el estudio de la DGA (2004b), para el análisis hidrológico se utilizaron dos estaciones con un régimen cercano al natural, que fueron obtenidas del Banco Nacional de Aguas (DGA, 2017a): (a) estación Sauces, para las subcuencas cordilleranas, pues presentan régimen mixto (códigos A, B, C y G en la Figura 2); y (b) estación Cholguán, a fin de determinar la disponibilidad en subcuencas con régimen pluvial (códigos D, E, F y G en la Figura 2). Estos casos se consideraron de 
Ciencias $₫$ Ägua
2020, Instituto Mexicano de Tecnología del Agua

Open Access bajo la licencia CC BY-NC-SA 4.0

(https://creativecommons.org/licenses/by-nc-sa/4.0/)

régimen natural, porque aguas arriba de su ubicación, dichas estaciones no registran extracciones de caudal.

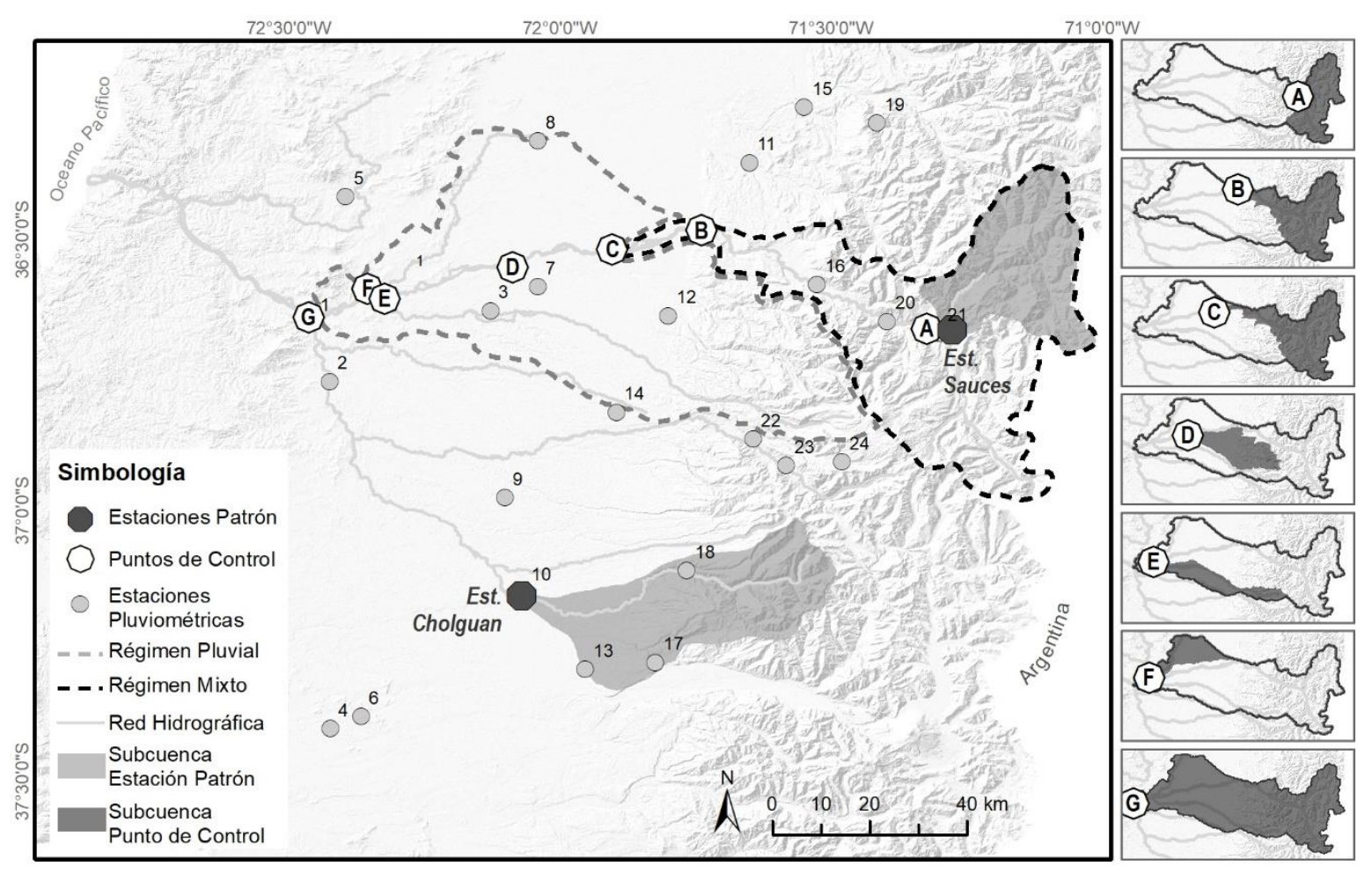

Figura 2. Estaciones, puntos y regímenes del área de estudio. En el panel principal se puede apreciar la localización de las cuencas patrón del estero Cholguán y estero Sauces en relación con la cuenca del río Ñuble. Los paneles de los costados identifican las subcuencas asociadas con cada punto de control.

Un caso especial se da con el código G, la desembocadura del río Ñuble, pues como muestran la Tabla 2 y Figura 2, posee un régimen pluvial y mixto (pluvio-nival). Es por ello que este punto utilizó como 
Tecnología y

Ciencias $₫$ Agua
2020, Instituto Mexicano de Tecnología del Agua

Open Access bajo la licencia CC BY-NC-SA 4.0

(https://creativecommons.org/licenses/by-nc-sa/4.0/)

patrón a las dos estaciones del área de estudio. La zona de régimen mixto del punto $\mathrm{G}$ se consideró como la subcuenca del punto $\mathrm{C}$, que se utilizó como patrón en la estación Sauces. El resto de la cuenca se consideró como la zona de régimen pluvial, la cual utilizó como patrón a la estación Cholguán.

Tabla 2. Ubicación y regímenes de estaciones y puntos evaluados.

Fuente: elaboración propia con base en datos de DGA (2004) y DGA (2017a).

\begin{tabular}{|c|c|c|c|c|c|c|}
\hline \multicolumn{2}{|c|}{ Código } & \multirow[t]{2}{*}{ Ubicación } & \multicolumn{2}{|c|}{ Coordenadas } & \multirow{2}{*}{$\begin{array}{l}\text { Altitud } \\
\text { (msnm) }\end{array}$} & \multirow[t]{2}{*}{ Régimen } \\
\hline & & & $\mathbf{S}$ & $\mathbf{W}$ & & \\
\hline \multirow{2}{*}{$\begin{array}{l}\text { Estación } \\
\text { Patrón }\end{array}$} & Sauces & Río Sauces antes junta con Ñuble & $36.666^{\circ}$ & $71.274^{\circ}$ & 700 & Mixto \\
\hline & Cholguán & Río Itata en Cholguán & $37.157^{\circ}$ & $72.068^{\circ}$ & 225 & Pluvial \\
\hline \multirow{7}{*}{$\begin{array}{c}\text { Punto } \\
\text { evaluado }\end{array}$} & $\bar{A}$ & Ñuble alto & $36.664^{\circ}$ & $71.322^{\circ}$ & 655 & Mixto \\
\hline & B & Ñuble medio & $36.483^{\circ}$ & $71.737^{\circ}$ & 288 & Mixto \\
\hline & $\mathrm{C}$ & Nuble medio & $36.519^{\circ}$ & $71.902^{\circ}$ & 191 & Mixto \\
\hline & $\mathrm{D}$ & Desembocadura río Cato & $36.552^{\circ}$ & $72.086^{\circ}$ & 114 & Pluvial \\
\hline & $\bar{E}$ & Desembocadura río Chillán & $36.601^{\circ}$ & $72.332^{\circ}$ & 53 & Pluvial \\
\hline & $\mathrm{F}$ & Desembocadura río Changaral & $36.601^{\circ}$ & $72.344^{\circ}$ & 49 & Pluvial \\
\hline & G & Desembocadura río Ñuble & $36.643^{\circ}$ & $72.462^{\circ}$ & 27 & Mixto / Pluvial \\
\hline
\end{tabular}

Se utilizaron las series de tiempo de los caudales medios mensuales de los últimos 25 años, es decir, a partir del año hidrológico de 1991-92 a 2016-17. El inicio del periodo se seleccionó debido a que 
presentó características normales de precipitación, pues según datos de la National Oceanic and Atmospheric Administration (NOAA, 2018), el fenómeno de El Niño en esa fecha no poseía valores extremos.

Se obtuvieron las probabilidades de excedencia (PE) de cada mes utilizando el software de análisis estadístico FRECwin, el cual determina dichas PE para cinco distribuciones de frecuencia de caudales (Weibull, Normal, Log-Normal, Gumbel, Gamma) y mediante el test de chi cuadrada se estimó la de mejor ajuste en cada caso, con el fin de obtener los caudales medios mensuales Q10PE, Q85PE y Q95PE (DGA, 2004a).

\section{Demanda hídrica}

Para el cálculo de la demanda se analizaron los DdA otorgados en el área de estudio, pues el caudal disponible depende de la diferencia entre el caudal existente en el río, menos los derechos que deben ser respetados y la demanda ambiental (DGA, 2008).

Se realizó un análisis de la totalidad de los DdA registrados hasta marzo de 2017 tanto en el Catastro Público de Aguas (CPA) obtenidos de la DGA (2017b), el cual corresponde a la base de datos oficial de DdA (DGA, 2008), como en una base de datos interna del Centro de Información de Recursos Hídricos (CIRH). El registro del $\mathrm{CIRH}$ 
Tecnología y

Ciencias $₫$ Agua
2020, Instituto Mexicano de Tecnología del Agua

Open Access bajo la licencia CCBY-NC-SA 4.0

(https://creativecommons.org/licenses/by-nc-sa/4.0/)

corresponde a DdA solicitados, pero no necesariamente constituidos $u$ otorgados por la DGA. En otras palabras, el dato de caudales de los DdA incluidos en esta base de datos es menos confiable. Para subsanar el problema anterior, se hizo una revisión de la totalidad de expedientes de DdA de la base $\mathrm{CIRH}$, con el objetivo de verificar el estado de constitución y el caudal otorgado.

Se encontraron 833 DdA en el área de estudio. Se eliminaron 101 DdA por no estar constituidos en litros por segundo, así como otros 88 DdA, porque se comprobó mediante la revisión de su expediente que su constitución fue denegada. En consecuencia, se utilizaron 644 DdA para el análisis.

Se detectó que no existen registros de algunos expedientes presentes en la base CIRH. Debido a lo anterior, se generaron dos escenarios con distintas distribuciones de caudales. El objetivo de esto fue evaluar cómo la inexactitud de una base de datos puede afectar la disponibilidad hídrica.

- Escenario 1: se consideraron los caudales de los DdA de la base oficial CPA y aquellos de la base CIRH que mediante la revisión de su expediente se verificaron.

- Escenario 2: se consideraron los caudales de los DdA del escenario 1 , sumado a aquellos de la base $\mathrm{CIRH}$ en que su expediente no pudo ser encontrado, por lo que no se tiene certeza de su constitución. 
Al obtener la totalidad de los caudales del área se hizo la suma de éstos en cada subcuenca, lo cual permitió conocer los valores de la demanda hídrica para cada escenario planteado.

\section{Balance hídrico}

Se efectuó el balance hídrico de los puntos evaluados según la norma establecida por la DGA (2008). El caudal disponible de ejercicio permanente $\left(Q_{\text {permanente }}\right)$, corresponde al caudal con PE de $85 \%$ (Q85PE), menos el caudal ecológico $\left(Q_{\text {ecológico }}\right)$, menos el caudal de $\operatorname{DdA}$ de ejercicio permanente ya constituidos $\left(Q_{c p}\right)$ según la Ecuación (2):

$$
Q_{\text {permanente }}=Q 85 P E-Q_{\text {ecológico }}-Q_{c p}
$$

A su vez, el caudal ecológico se considera como $50 \%$ del caudal con probabilidad de excedencia de 95\% (50Q95PE), con las restricciones mencionadas en el Decreto $\mathrm{N}^{\circ} 71$ (Ministerio del Medio Ambiente, 2015), y que se indican a continuación: 
- Para los meses en que el 50Q95PE es menor al caudal determinado para $20 \%$ del caudal medio anual (20Qma), el caudal ecológico será 50Q95PE.

- Para los meses en que 50Q95PE es mayor que el caudal determinado para 20Qma, el caudal ecológico será 20Qma.

Para los caudales disponibles de ejercicio eventual ( $\left.Q_{\text {eventual }}\right)$, se utilizaron según la Ecuación (3), el Q10PE menos el Q85PE, menos el caudal de DdA de ejercicio eventual ya constituidos $\left(Q_{c e}\right)$, menos el posible déficit de recursos de ejercicio permanente $\left(Q_{d p}\right)$ que se genere del análisis de disponibilidad (DGA, 2008). Así:

$Q_{\text {eventual }}=Q 10 P E-Q 85 P E-Q_{c e}-Q_{d p}$

\section{Resultados}

\section{Determinación de precipitaciones}


2020, Instituto Mexicano de Tecnología del Agua

Open Access bajo la licencia CC BY-NC-SA 4.0

(https://creativecommons.org/licenses/by-nc-sa/4.0/)

El sesgo entre las precipitaciones del TRMM y las observaciones instrumentales alcanzó diferencias de hasta 91.5\%. Dado que la distribución espacial de las estaciones es irregular, se optó por utilizar el TRMM para producir mapas de precipitación. Sin embargo, la comparación entre la precipitación de TRMM y las estaciones entregó un sesgo de $33.9 \%$. Para reducir este sesgo se realizó una corrección de los montos de cada celda del TRMM que cubren el área de estudio.

Se encontró una relación entre la altitud de la estación y el sesgo de precipitaciones presente con respecto al TRMM, ya que como se observa en la Figura 3, a mayor altitud de la estación se genera mayor sesgo. Debido a esto, se ejecutó una corrección a partir de un modelo de regresión lineal entre las variables mencionadas. Al incluir en el modelo a la estación Las Trancas (Tabla 1 ), se obtuvo un $R^{2}$ (Ecuación (5)) bajo, por lo que se optó por eliminarla de este cálculo. La estación Las Trancas es la más alta del área de estudio y está situada en una zona encajonada, donde gran parte de la precipitación invernal corresponde a nieve, a diferencia del resto de las estaciones de la zona, que se localizan bajo la línea de nieve determinada para cuencas de esa región (Stehr \& Aguayo, 2017). Ya que TRMM sólo estima precipitación líquida, es muy posible que la falta de correlación se pueda explicar por esto. Así, se obtuvo un modelo que explica un $84 \%$ de la varianza. 
Tecnología y

Ciencias $\sqrt[\triangleleft]{\triangleleft}$ gua
2020, Instituto Mexicano de Tecnología del Agua

Open Access bajo la licencia CCBY-NC-SA 4.0

(https://creativecommons.org/licenses/by-nc-sa/4.0/)

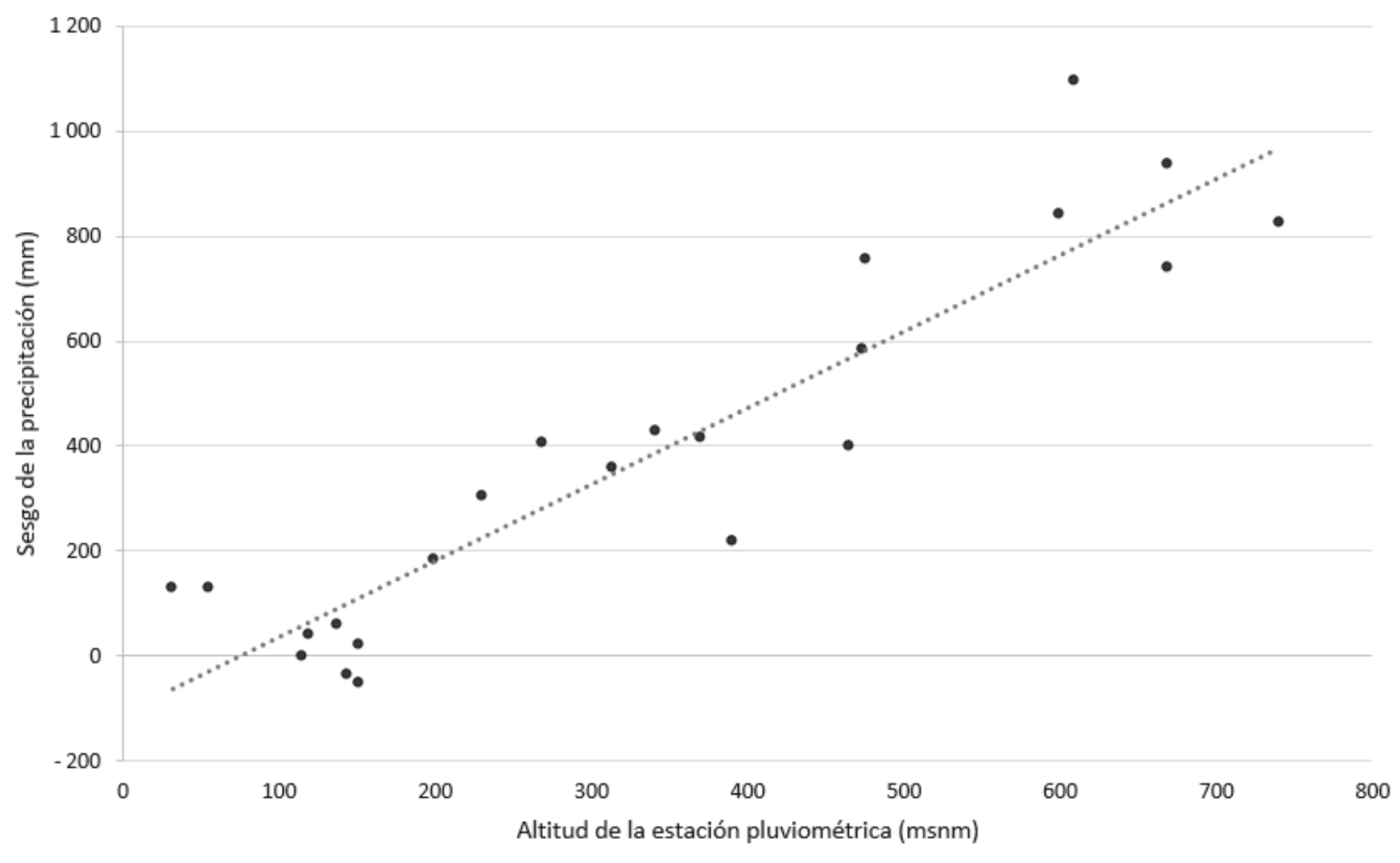

Figura 3. Modelo de regresión lineal.

A continuación, la Ecuación (4) de ajuste de la Figura 3:

$y=1.454 x-108.18-R^{2}=0.8433$

Con la Ecuación (4) se obtuvieron los valores del sesgo que debería tener cada celda, según la altitud que posee. Estos valores se adicionaron al TRMM, logrando un ajuste que generó un sesgo promedio de un $14.8 \%$; con esto, el sesgo se redujo a menos de la mitad del 
Tecnología y

Ciencias $₫$ Agua
2020, Instituto Mexicano de Tecnología del Agua

Open Access bajo la licencia CC BY-NC-SA 4.0

(https://creativecommons.org/licenses/by-nc-sa/4.0/)

original. Por otro lado, cabe destacar que si bien en algunas estaciones de baja altitud el sesgo aumentó, en aquellas de mayor elevación la disminución fue muy notoria. Lo anterior se observa en la Figura 4, que muestra la distribución de las precipitaciones. El número presente corresponde al código de las estaciones (Tabla 1).

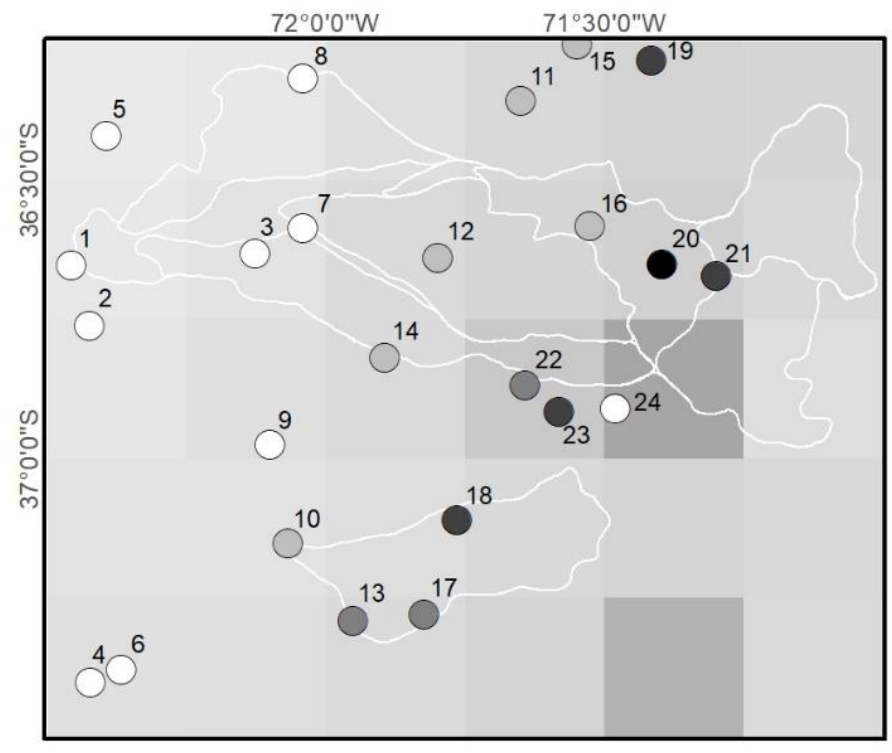

TRMM Original
Precipitación

$\operatorname{TRMM}(\mathrm{mm}) \quad 500$

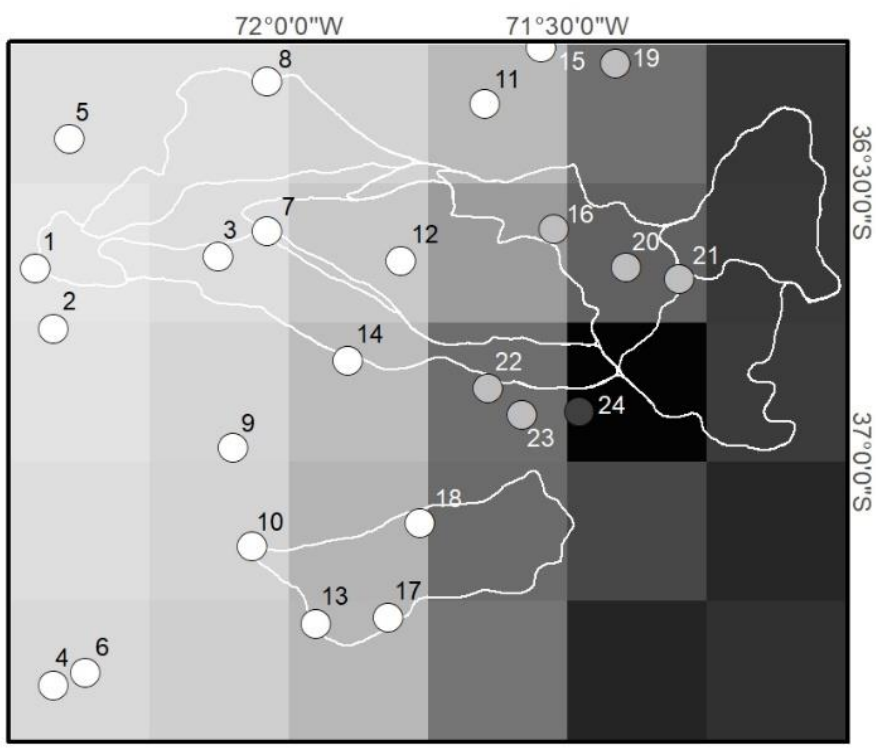

TRMM Corregido

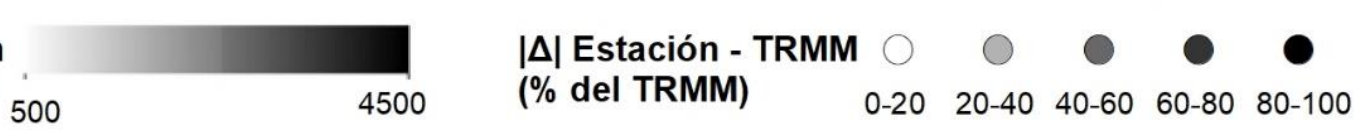

Figura 4. Precipitación TRMM y sesgos con estaciones pluviométricas.

A partir del modelo corregido, se calculó la precipitación media anual de las subcuencas de estudio y de las estaciones patrón, las cuales se muestran en la Tabla 3, junto con el área correspondiente a cada una. El punto G* atañe a la zona de régimen pluvial de la cuenca 
Tecnología y

Ciencias $₫$ Agua
2020, Instituto Mexicano de Tecnología del Agua

Open Access bajo la licencia CC BY-NC-SA 4.0

(https://creativecommons.org/licenses/by-nc-sa/4.0/)

del río Ñuble que, sumado al punto $\mathrm{C}$, da como resultado el área completa de esta cuenca (ver Figura 2 y Tabla 2 ).

Tabla 3. Área y precipitación por subcuencas.

\begin{tabular}{|c|c|c|c|c|c|c|c|c|c|}
\hline & \multicolumn{2}{|c|}{ Estación patrón } & \multicolumn{6}{c|}{ Punto evaluado } \\
\cline { 2 - 10 } & Sauces & Cholguán & A & B & C & D & E & F & G* \\
\hline Área $\left(\mathrm{km}^{2}\right)$ & 607 & 839 & 1257 & 1879 & 1929 & 1015 & 779 & 705 & 2958 \\
\hline $\begin{array}{c}\text { Precipitación } \\
\text { (mm/año })\end{array}$ & 3534 & 2176 & 3667 & 3357 & 3303 & 1838 & 1739 & 1002 & 1487 \\
\hline
\end{tabular}

\section{Oferta hídrica}

Mediante la trasposición de cuencas se obtuvieron los caudales en régimen natural presentes en la Tabla 4. Los puntos ubicados sobre el río Ñuble son los que presentaron mayor caudal, destacando la desembocadura de éste con valores sobre los $150 \mathrm{~m}^{3} / \mathrm{s}$ a $85 \mathrm{PE}$.

Tabla 4. Puntos evaluados con Q10PE, Q85PE y Q95PE por meses. Las distribuciones de probabilidad que se ajustaron mejor a cada lugary mes se representan con tonos de grises, con su leyenda incluida al fondo de la tabla. 
Tecnología

Ciencias $₫$ Agua
2020, Instituto Mexicano de Tecnología del Agua

Open Access bajo la licencia CC BY-NC-SA 4.0

(https://creativecommons.org/licenses/by-nc-sa/4.0/)

\begin{tabular}{|c|c|c|c|c|c|c|c|c|c|c|c|c|c|}
\hline \multirow[t]{2}{*}{ Código } & \multirow[t]{2}{*}{ PE } & \multicolumn{12}{|c|}{ Caudal $\left(\mathrm{m}^{3} / \mathrm{s}\right)$} \\
\hline & & Abr & May & Jun & Jul & $\overline{\text { Ago }}$ & Sep & Oct & Nov & Dic & Ene & Feb & Mar \\
\hline \multirow[t]{3}{*}{ Sauces } & 10 & 16 & 62 & 97 & 72 & 79 & 74 & 75 & 71 & 46 & 23 & 13 & 11 \\
\hline & 85 & 4 & 6 & 21 & 26 & 22 & 32 & 33 & 27 & 14 & 6 & 4 & 4 \\
\hline & 95 & 3 & 3 & 14 & 19 & 16 & 25 & 23 & 15 & 5 & 4 & 3 & 3 \\
\hline \multirow[t]{3}{*}{ Cholguán } & 10 & 25 & 94 & 134 & 133 & 118 & 94 & 68 & 40 & 29 & 23 & 24 & 21 \\
\hline & 85 & 10 & 9 & 38 & 36 & 36 & 36 & 25 & 21 & 16 & 14 & 14 & 12 \\
\hline & 95 & 8 & 5 & 23 & 11 & 15 & 28 & 18 & 18 & 13 & 13 & 12 & 11 \\
\hline \multirow[t]{3}{*}{ A } & 10 & 34 & 133 & 209 & 154 & 170 & 159 & 161 & 152 & 100 & 49 & 29 & 23 \\
\hline & 85 & 9 & 13 & 44 & 56 & 48 & 69 & 72 & 57 & 29 & $\overline{14}$ & 10 & 8 \\
\hline & 95 & 6 & 7 & 29 & 41 & 34 & 55 & 49 & 32 & 11 & 9 & 7 & 6 \\
\hline \multirow[t]{3}{*}{$B$} & 10 & 47 & 182 & 286 & 211 & 233 & 218 & 220 & 209 & 136 & 66 & 39 & 31 \\
\hline & 85 & 12 & 18 & 61 & 77 & 65 & 94 & 98 & 78 & 40 & 19 & 13 & 11 \\
\hline & 95 & 8 & 10 & 40 & 56 & 47 & 75 & 66 & 44 & 15 & 12 & 9 & 8 \\
\hline \multirow[t]{3}{*}{$\mathrm{C}$} & 10 & 48 & 184 & 289 & 214 & 236 & 220 & 223 & 211 & 138 & 67 & 40 & 32 \\
\hline & 85 & 12 & 19 & 61 & 78 & 66 & 95 & 99 & 79 & 40 & 19 & 13 & 11 \\
\hline & 95 & 8 & 10 & 41 & 56 & 47 & 76 & 67 & 45 & 15 & 12 & 9 & 8 \\
\hline \multirow[t]{3}{*}{$\mathrm{D}$} & 10 & 26 & 96 & 137 & 136 & 121 & 96 & 69 & 41 & 29 & 23 & 24 & 22 \\
\hline & 85 & 10 & 9 & 39 & 37 & 37 & 37 & 25 & 21 & 17 & 15 & 14 & 13 \\
\hline & 95 & 8 & 5 & 23 & 11 & 15 & 29 & 19 & 18 & 13 & 13 & 12 & 11 \\
\hline \multirow[t]{3}{*}{$\bar{E}$} & 10 & 19 & 70 & 99 & 99 & 88 & 70 & 50 & 30 & 21 & 17 & 18 & 16 \\
\hline & 85 & 8 & 7 & 28 & 27 & 27 & 27 & 18 & 16 & 12 & 11 & 10 & 9 \\
\hline & 95 & 6 & 4 & 17 & 8 & 11 & 21 & 14 & 13 & 10 & 10 & 9 & 8 \\
\hline $\mathrm{F}$ & 10 & 10 & 36 & 52 & 51 & 46 & 36 & 26 & 15 & 11 & 9 & 9 & 8 \\
\hline
\end{tabular}


Tecnología y

Ciencias ฐึgua
2020, Instituto Mexicano de Tecnología del Agua

Open Access bajo la licencia CC BY-NC-SA 4.0

(https://creativecommons.org/licenses/by-nc-sa/4.0/)

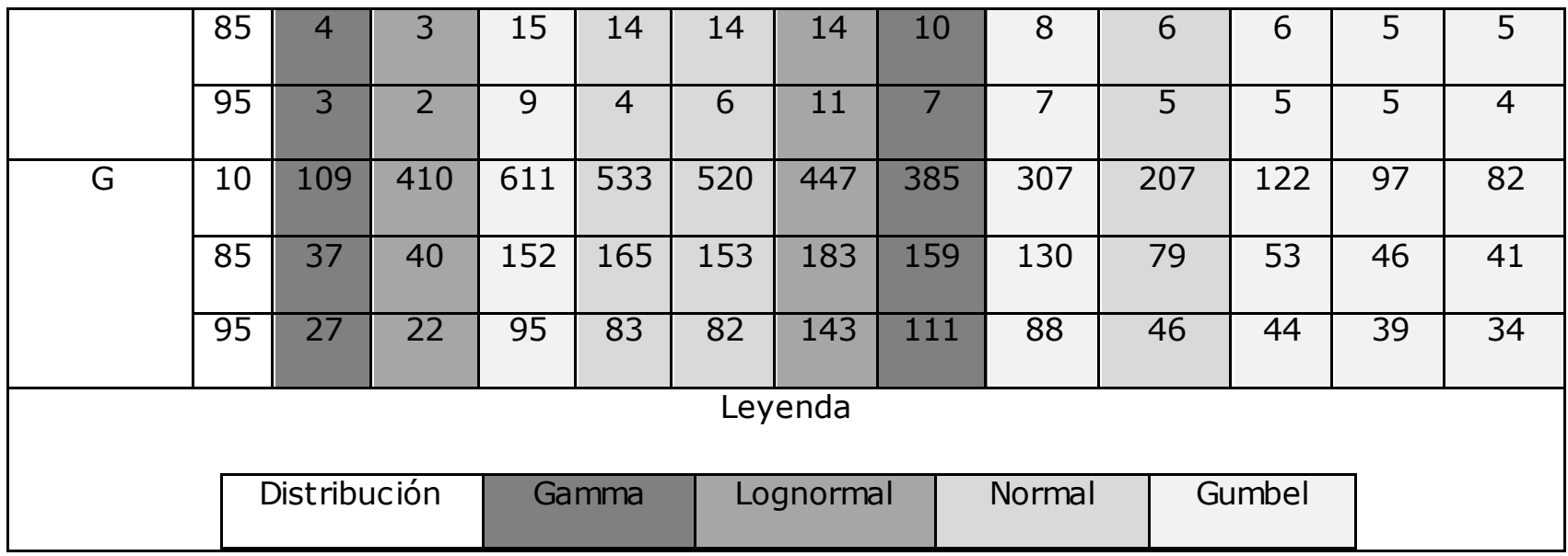

Además, a partir de los Qma de las estaciones, mediante el uso de la Ecuación (1) (transposición de cuencas), se calcularon los Qma de los puntos evaluados (Tabla 5). Se observa una gran diferencia entre el río Ñuble y los demás, ya que, por ejemplo, la desembocadura del río Changaral (F) sólo posee alrededor del $8 \%$ del caudal del río Ñuble (G).

Tabla 5. Caudal medio anual (Qma) por puntos evaluados.

\begin{tabular}{|c|c|c|c|c|c|c|c|c|c|}
\hline & \multicolumn{6}{|c|}{ Estación patrón } & \multicolumn{6}{|c|}{ Punto evaluado } \\
\cline { 2 - 10 } & Sauces & Cholguán & A & B & C & D & E & F & G \\
\hline Caudal medio anual $\left(\mathrm{m}^{3} / \mathrm{s}\right)$ & 31.8 & 41.3 & 68.3 & 93.4 & 94.4 & 42.3 & 30.7 & 16 & 194 \\
\hline
\end{tabular}

\section{Demanda hídrica}


Tecnología y

Ciencias $₫$ Agua
2020, Instituto Mexicano de Tecnología del Agua

Open Access bajo la licencia CC BY-NC-SA 4.0

(https://creativecommons.org/licenses/by-nc-sa/4.0/)

La distribución de caudales entre derechos de ejercicio permanente y eventual tuvo diferencias. Por un lado, los derechos permanentes poseen una distribución más homogénea durante todos los meses del año, es decir, los caudales otorgados no tienen mucha variabilidad mensual. Por otro lado, los derechos eventuales sí poseen gran variabilidad, alcanzando diferencias de hasta $134 \mathrm{~m}^{3} / \mathrm{s}$ (Tabla 6) entre los meses de invierno y verano, en donde el caudal otorgado es mucho mayor en invierno.

Tabla 6. Caudal otorgado por subcuenca de puntos evaluados.

\begin{tabular}{|c|c|c|c|c|c|c|c|c|c|c|c|c|c|}
\hline \multirow{2}{*}{ Derechos } & \multirow{2}{*}{ Punto } & \multicolumn{12}{|c|}{ Caudal $\left(\mathrm{m}^{3} / \mathrm{s}\right)$} \\
\hline & & $\mathbf{A b r}$ & May & Jun & Jul & Ago & Sep & Oct & Nov & Dic & Ene & Feb & Mar \\
\hline \multirow{7}{*}{ 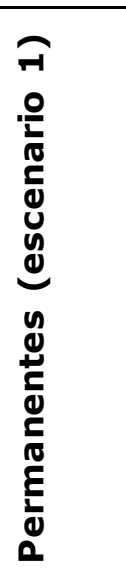 } & A & 0 & 0 & 0 & 0 & 0 & 0 & 0 & 0 & 0 & 0 & 0 & 0 \\
\hline & $B$ & 5.5 & 5.5 & 5.5 & 5.5 & 5.5 & 5.5 & 5.5 & 5.5 & 5.5 & 5.5 & 5.5 & 5.5 \\
\hline & C & 12.9 & 12.9 & 12.9 & 12.9 & 12.9 & 12.9 & 12.9 & 12.9 & 12.9 & 12.9 & 12.9 & 12.9 \\
\hline & $\mathrm{D}$ & 6.2 & 6.9 & 7 & 7 & 7 & 6.9 & 6.3 & 6.2 & 6.2 & 6.2 & 6.2 & 6.2 \\
\hline & $E$ & 4.4 & 7.4 & 7.5 & 7.5 & 7.5 & 7.5 & 4.5 & 4.5 & 4.4 & 4.2 & 4.4 & 4.4 \\
\hline & $\mathrm{F}$ & 1.2 & 1.2 & 1.2 & 1.2 & 1.2 & 1.2 & 1.2 & 1.2 & 1.2 & 1.2 & 1.2 & 1.2 \\
\hline & G & 25.7 & 29.4 & 29.5 & 29.5 & 29.5 & 29.5 & 25.9 & 25.8 & 25.8 & 25.6 & 25.7 & 25.7 \\
\hline \multirow{5}{*}{ 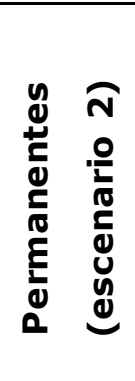 } & $A$ & 0 & 0 & 0 & 0 & 0 & 0 & 0 & 0 & 0 & 0 & 0 & 0 \\
\hline & $B$ & 6.3 & 6.3 & 6.3 & 6.3 & 6.3 & 6.3 & 6.3 & 6.3 & 6.3 & 6.3 & 6.3 & 6.3 \\
\hline & $C$ & 14.5 & 14.5 & 14.5 & 14.5 & 14.5 & 14.5 & 14.5 & 14.5 & 14.5 & 14.5 & 14.5 & 14.5 \\
\hline & $\mathrm{D}$ & 7.1 & 7.7 & 7.8 & 7.8 & 7.8 & 7.8 & 7.2 & 7.1 & 7.1 & 7.1 & 7.1 & 7.1 \\
\hline & $E$ & 5.4 & 8.4 & 8.4 & 8.4 & 8.4 & 8.4 & 5.4 & 5.4 & 5.4 & 5.2 & 5.4 & 5.4 \\
\hline
\end{tabular}


Tecnología y

Ciencias $₫$ Agua
2020, Instituto Mexicano de Tecnología del Agua

Open Access bajo la licencia CCBY-NC-SA 4.0

(https://creativecommons.org/licenses/by-nc-sa/4.0/)

\begin{tabular}{|c|c|c|c|c|c|c|c|c|c|c|c|c|c|}
\hline & $F$ & 2.1 & 2.1 & 2.1 & 2.1 & 2.1 & 2.1 & 2.1 & 2.1 & 2.1 & 2.1 & 2.1 & 2.1 \\
\hline & $\bar{G}$ & 30.9 & 34.6 & 34.7 & 34.7 & 34.7 & 34.7 & 31.1 & 31 & 30.9 & 30.7 & 30.9 & 30.9 \\
\hline ล & $A$ & 19 & 69.9 & 111.8 & 71.1 & 54.4 & 56.7 & 83.1 & 149.6 & 86.6 & 19 & 19 & 19 \\
\hline$\vec{r}$ & $B$ & 63.3 & 114.2 & 156 & 115.4 & 98.6 & 100.9 & 127.3 & 193.9 & 130.8 & 63.3 & 63.3 & 63.3 \\
\hline$\underbrace{2}_{\pi}$ & $\bar{C}$ & 63.3 & 114.2 & 156 & 115.4 & 98.6 & 100.9 & 127.3 & 193.9 & 130.8 & 63.3 & 63.3 & 63.3 \\
\hline ü & $\bar{D}$ & 0 & 0.1 & $\overline{0}$ & 0 & 0 & $\overline{0}$ & $\overline{0}$ & 0.1 & 0.1 & 0 & 0 & 0 \\
\hline$\vec{n}$ & $\mathrm{E}$ & 1.3 & 6.5 & 15.9 & 19.3 & 14.7 & 12.9 & 6.3 & 4 & 2 & 0.7 & 0.7 & 0.7 \\
\hline$\frac{\pi}{3}$ & $F$ & 0 & 2.2 & 2.2 & 2.2 & 2.2 & 2.2 & 0 & 0 & 0 & 0 & 0 & 0 \\
\hline 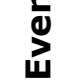 & $\mathrm{G}$ & 64.6 & 123.1 & 174.2 & 137 & 115.6 & 116.1 & 133.6 & 198 & 132.9 & 63.9 & 63.9 & 63.9 \\
\hline
\end{tabular}

El escenario 2 da cuenta de que la inexactitud de una base de datos puede generar diferencias de caudales otorgados de hasta 5.2 $\mathrm{m}^{3} / \mathrm{s}$. Este es el caso del Punto $G$, que posee caudales permanentes verificados que oscilan entre 25 y $30 \mathrm{~m}^{3} / \mathrm{s}$ (escenario 1), mientras que si se consideran los DdA que no pudieron ser verificados (escenario 2), se alcanzan caudales concedidos de hasta $34.7 \mathrm{~m}^{3} / \mathrm{s}$.

\section{Balance hídrico}


Tecnología y

Ciencias $₫$ Agua
2020, Instituto Mexicano de Tecnología del Agua

Open Access bajo la licencia CC BY-NC-SA 4.0

(https://creativecommons.org/licenses/by-nc-sa/4.0/)

El balance hecho arrojó resultados similares en ambos escenarios, existiendo déficit entre los meses de octubre a mayo, por lo que en un año seco no se podría satisfacer el ejercicio de la totalidad de los DdA.

La Tabla 7 presenta la disponibilidad en los puntos evaluados. En negativo se presentan los caudales sobreotorgados, donde la demanda es mayor que la oferta hídrica. Además, se presentan con el símbolo * aquellos caudales que pasaron de estar en calidad de disponibles en el escenario 1, a sobreotorgados en el escenario 2.

Tabla 7. Disponibilidad de caudal por punto evaluado.

\begin{tabular}{|c|c|c|c|c|c|c|c|c|c|c|c|c|c|c|}
\hline \multirow{2}{*}{\multicolumn{2}{|c|}{$\begin{array}{l}\text { Disponi- } \\
\text { bilidad }\end{array}$}} & \multirow{3}{*}{\begin{tabular}{|c|}
$\begin{array}{c}\text { Pun } \\
\text { to }\end{array}$ \\
$\mathrm{A}$
\end{tabular}} & \multicolumn{12}{|c|}{ Caudal $\left(\mathrm{m}^{3} / \mathrm{s}\right)$} \\
\hline & & & Abr & May & Jun & Jul & Ago & Sep & Oct & Nov & Dic & Ene & Feb & Mar \\
\hline \multirow{11}{*}{ 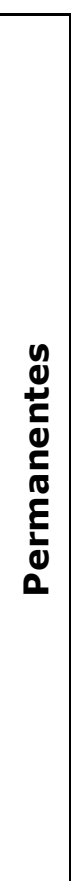 } & \multirow{7}{*}{ 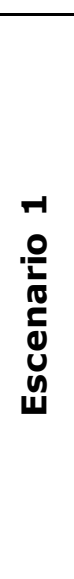 } & & 5.6 & 9.8 & 30.7 & 42.6 & 33.9 & 55.3 & 58.3 & 43.7 & 23.8 & 9.1 & 6.2 & 5.2 \\
\hline & & $B$ & 2.1 & 7.9 & 36.4 & 52.9 & 40.9 & 70.1 & 74.3 & 54.2 & 27.1 & 6.9 & 3 & 1.5 \\
\hline & & $\mathrm{C}$ & -5.1 & $0.7^{*}$ & 29.5 & 46.1 & 34 & 63.6 & 67.7 & 47.5 & 20.1 & -0.3 & -4.3 & -5.7 \\
\hline & & $\mathrm{D}$ & $0.3^{*}$ & -0.3 & 23.3 & 24.6 & 22.5 & 21.6 & 10.7 & 6.8 & 3.8 & 1.8 & 1.6 & $0.8^{*}$ \\
\hline & & $E$ & $0.3^{*}$ & -2.6 & 14.5 & 15.4 & 14 & 13.3 & 7.9 & 5 & 2.8 & 1.6 & 1.3 & $0.6^{*}$ \\
\hline & & $\mathrm{F}$ & 1.3 & 1.3 & 10.3 & 10.8 & 10 & 9.6 & 5.3 & 3.8 & 2.6 & 1.9 & 1.8 & 1.5 \\
\hline & & $\mathrm{G}$ & -2.6 & -0.3 & 84.1 & 97.1 & 84.6 & 114.2 & 94.8 & 65.2 & 30.6 & 5.9 & $1.3^{*}$ & -2.2 \\
\hline & \multirow{4}{*}{ 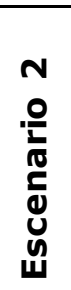 } & $A$ & 5.6 & 9.7 & 30.6 & 42.6 & 33.9 & 55.3 & 58.3 & 43.6 & 23.8 & 9.1 & 6.2 & 5.1 \\
\hline & & $B$ & 1.4 & 7.1 & 35.7 & 52.1 & 40.1 & 69.4 & 73.5 & 53.5 & 26.3 & 6.2 & 2.2 & 0.8 \\
\hline & & $\bar{C}$ & -6.7 & $-1^{*}$ & 27.9 & 44.5 & 32.4 & 61.9 & 66.1 & 45.9 & 18.4 & -1.9 & -5.9 & -7.4 \\
\hline & & $\mathrm{D}$ & $-0.6^{*}$ & -1.1 & 22.4 & 23.7 & 21.7 & 20.8 & 9.8 & 5.9 & 2.9 & 1 & 0.8 & $-0.1^{*}$ \\
\hline
\end{tabular}


Tecnología y

Ciencias $₫$ Agua
2020, Instituto Mexicano de Tecnología del Agua

Open Access bajo la licencia CCBY-NC-SA 4.0

(https://creativecommons.org/licenses/by-nc-sa/4.0/)

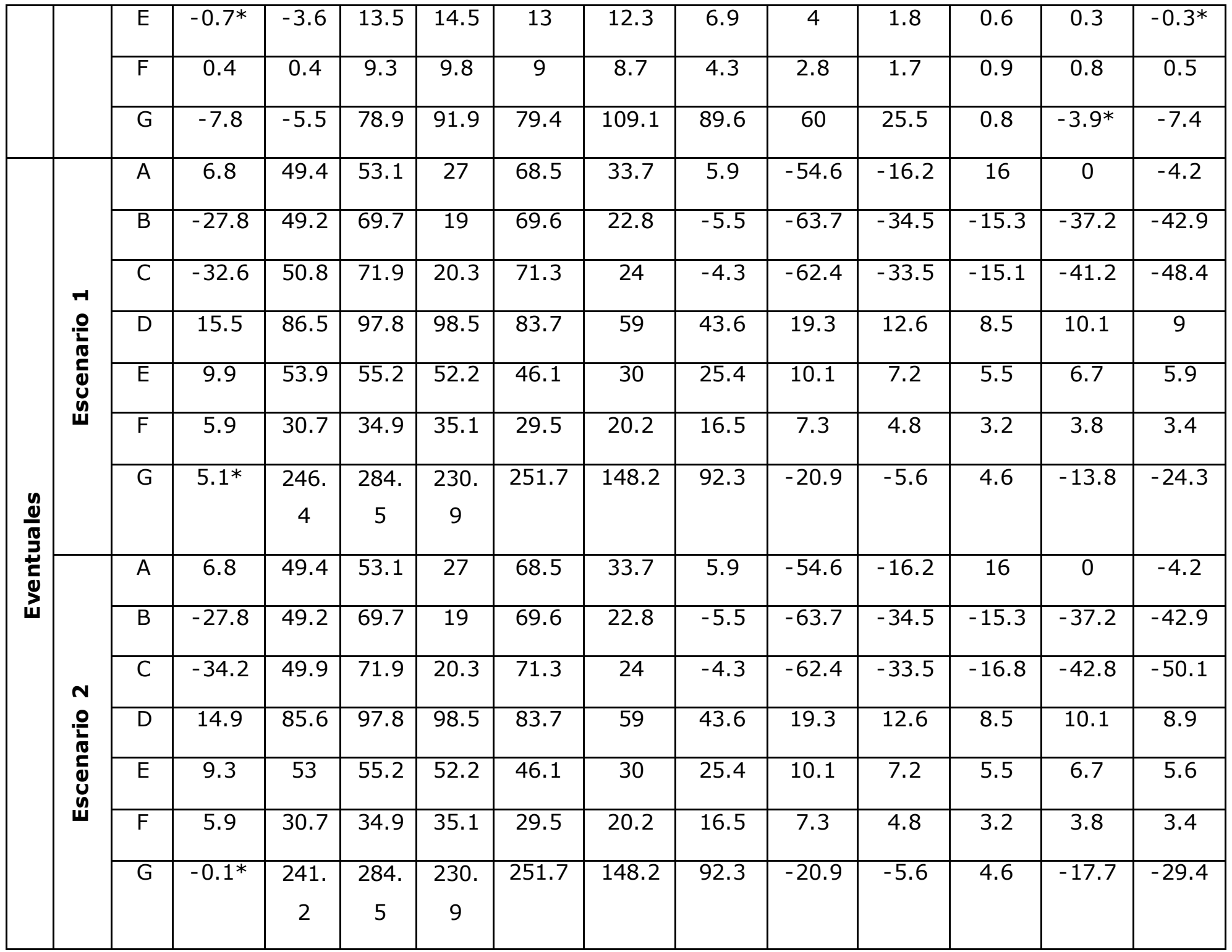

El planteamiento del escenario 2, si bien no significa gran diferencia con respecto al 1 , en ciertos puntos genera un sobreotorgamiento que en el primer escenario no existía, principalmente en derechos de ejercicio permanente. El cambio más drástico es en el punto $G$ en el mes de febrero, donde en el escenario 1 hay 
Ciencias $₫$ Agua
2020, Instituto Mexicano de Tecnología del Agua

Open Access bajo la licencia CC BY-NC-SA 4.0

(https://creativecommons.org/licenses/by-nc-sa/4.0/)

disponibilidad de $1.3 \mathrm{~m}^{3} / \mathrm{s}$ y en el escenario 2 se produce un déficit de $3.9 \mathrm{~m}^{3} / \mathrm{s}$. Esta situación también se genera en los ríos Cato y Chillán (puntos $D$ y E) durante los meses de marzo y abril, donde en el escenario 1 existe disponibilidad de más de $0.3 \mathrm{~m}^{3} / \mathrm{s}$ y en el escenario 2 el recurso se ve agotado, con un déficit de hasta $0.7 \mathrm{~m}^{3} / \mathrm{s}$.

Los principales déficits para el caso de los derechos permanentes, considerando sólo el escenario 2, se observan en los puntos C y G, entre los meses de enero a mayo (Tabla 7 y Figura 5), con máximos que superan los $7 \mathrm{~m}^{3} / \mathrm{s}$.

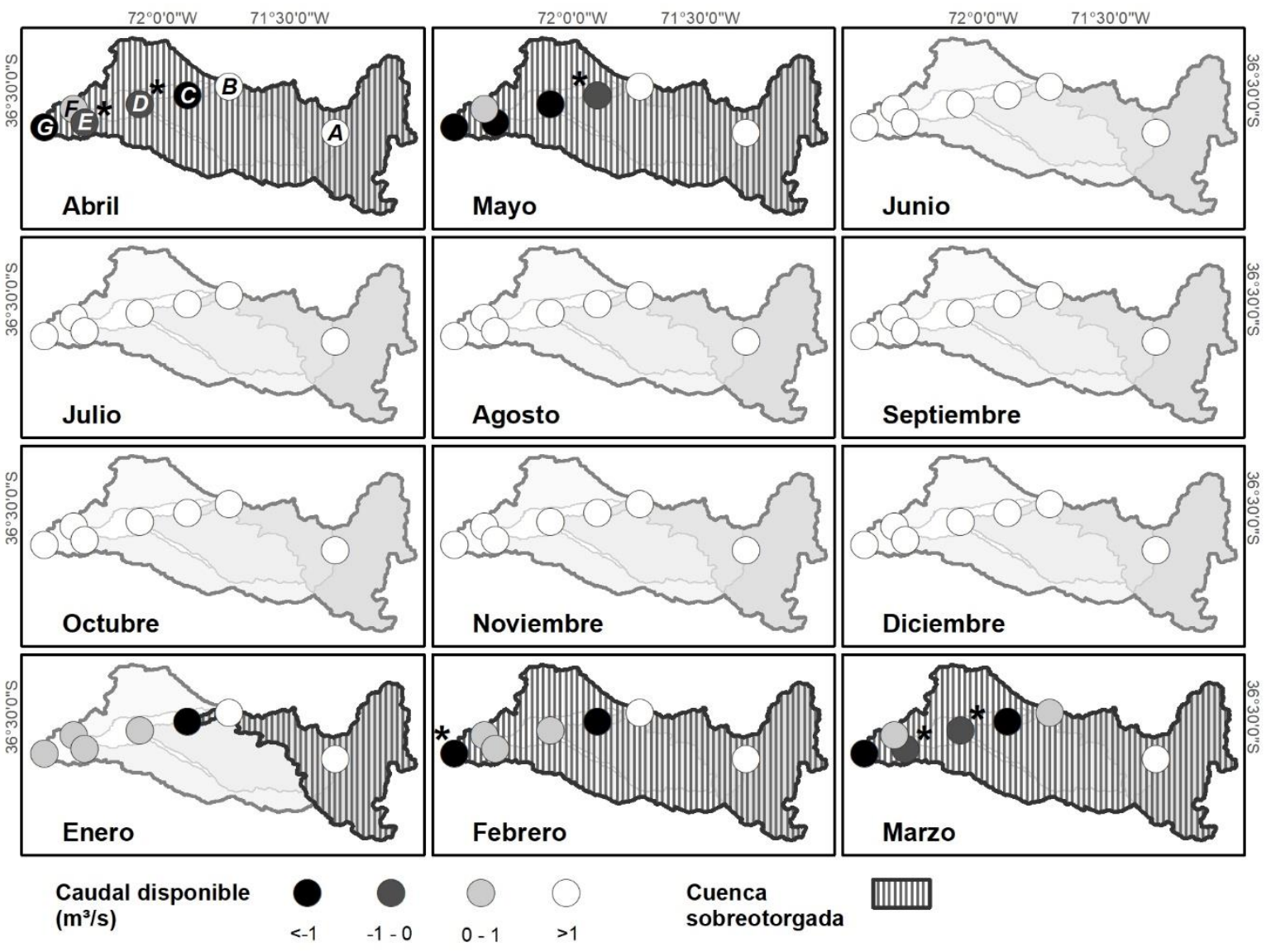


Figura 5. Disponibilidad de caudal permanente para el escenario 2.

Al considerar la disponibilidad global de la cuenca del río Ñuble, se puede identificar al punto $\mathrm{G}$ como el más crítico, pues genera la caducidad de su área aportante. Esto es indicado por el área achurada de la Figura 6, en la que se observa que de febrero a mayo está sobreotorgada la cuenca, ya que a pesar de que existe disponibilidad aguas arriba (puntos $A, B$ y F), debe ser preservada para la satisfacción de los derechos existentes aguas abajo, lo cual impide la constitución de nuevos DdA. Además, en el mes de enero, la subcuenca del punto $C$ está sobreotorgada, por lo que en ésta tampoco se pueden constituir más derechos, a diferencia de los puntos ubicados aguas abajo ( $D, E, F$ y G), en que sí existe disponibilidad. 
Tecnología y

Ciencias $₫$ Agua
2020, Instituto Mexicano de Tecnología del Agua

Open Access bajo la licencia CC BY-NC-SA 4.0

(https://creativecommons.org/licenses/by-nc-sa/4.0/)

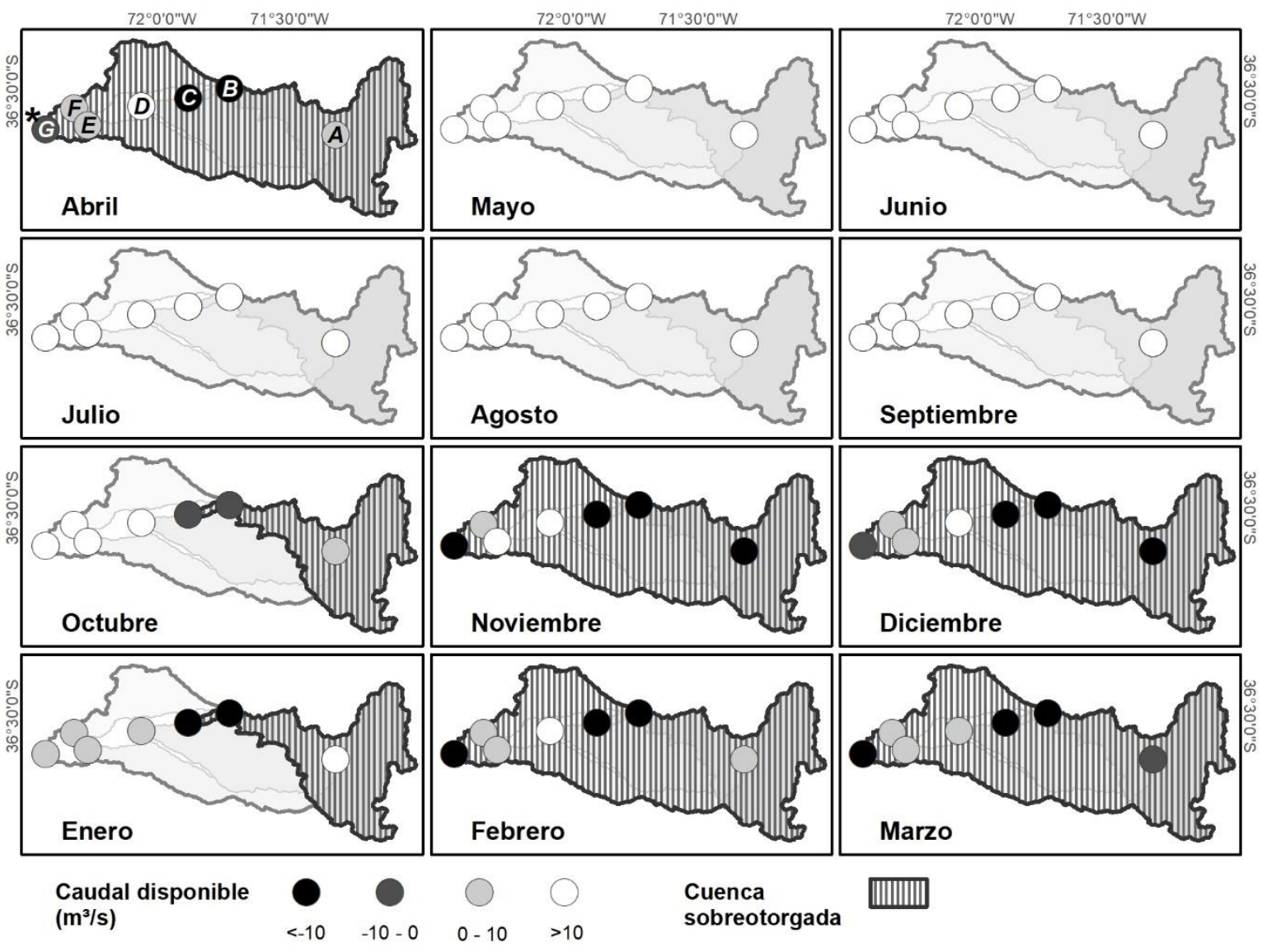

Figura 6. Disponibilidad de caudal eventual para el escenario 2.

La Figura 6 presenta una situación similar, pero abarca más meses, en donde la cuenca del río Ñuble se ve escasa desde noviembre hasta abril, sin considerar el mes de enero; el área del punto $C$ está en igual situación en los meses de enero y octubre.

Los principales déficits para el caso de los derechos eventuales en el escenario 2 ocurren en los puntos $B$ y $C$, entre los meses de octubre y abril, con máximos mayores a $60 \mathrm{~m}^{3} / \mathrm{s}$, en el mes de noviembre (Tabla 7 y Figura 6). Por otro lado, los puntos $D, E$ y $F$ poseen una 
disponibilidad local del recurso hídrico durante todo el año; pero aun así, en ciertos meses no se pueden otorgar derechos a causa del déficit existente aguas abajo.

Por lo tanto, los resultados indican que existe un sobreotorgamiento del recurso en meses de verano, tanto en el escenario 1, que incluyó todos los posibles derechos otorgados, como en el escenario 2, que consideró sólo los DdA que figuran en los registros DGA y CIRH como verificados y constituidos.

\section{Discusión}

La evaluación de la disponibilidad hídrica para la constitución de DdA en la cuenca del río Ñuble arrojó como resultado un contexto de sobreotorgamiento del recurso, en que no existe disponibilidad para DdA de ejercicio permanente desde febrero hasta mayo, y para DdA de ejercicio eventual en noviembre, diciembre, febrero, marzo y abril. El punto causante del sobreotorgamiento corresponde a la desembocadura del río Ñuble, el cual deja agotada toda la cuenca. Además, se observa un déficit en la parte alta de la cuenca en octubre y enero.

Estos hallazgos ponen en evidencia las ineficiencias de un sistema de concesión de derechos que ha producido una agudización del estrés 
hídrico y destrucción irreversible de cuencas en muchas regiones donde el recurso es escaso (Larraín, 2006).

La DGA no ha podido cumplir con su función de fiscalizar y catastrar los DdA (Bolados et al., 2017), provocando equivocaciones en el otorgamiento de DdA, lo cual se agrava en un contexto de cambio hacia un nuevo escenario hidrológico en que la existencia de episodios de sequía es cada vez más frecuente en la zona central de Chile (Muñoz et al., 2016).

Los problemas asociados con las dificultades en el control del sobreotorgamiento de DdA y la posibilidad de aumento en la demanda de agua pueden impactar con mayor fuerza en la disponibilidad de agua en el futuro, considerando las tendencias hidroclimáticas documentadas para las últimas décadas y las proyecciones. Evidencias de un nuevo escenario hidroclimático se documentan en el retroceso general de glaciares y aumento de la altitud de la línea de equilibrio (Carrasco, Osorio, \& Casassa, 2008); disminución de las precipitaciones (UrrutiaJalabert, Gonzáles, Gonzáles-Reyes, Lara, \& Garreaud, 2018); caudales por debajo del promedio (Fernández et al., 2018; Barría, Peel, Walsh, \& Muñoz, 2017), y la megasequía que comenzó en 2010 (CR2, 2015). De hecho, ha sido la sequía continua más larga de la que se tenga registro instrumental y la que ha llegado más al sur ( $\left.38^{\circ} \mathrm{S}\right)$, con un déficit anual de lluvia que va de un 25 a 45\% (Garreaud et al., 2017). Sin embargo, es importante destacar que dicha sequía meteorológica no necesariamente se transforma en una hidrológica, pues ésta depende de factores específicos de cada cuenca, en especial cobertura vegetal, tipos de suelo y morfometría (Fernandez et al., 2018). Las proyecciones 
Ciencias $₫$ Agua
2020, Instituto Mexicano de Tecnología del Agua

Open Access bajo la licencia CCBY-NC-SA 4.0

(https://creativecommons.org/licenses/by-nc-sa/4.0/)

climáticas basadas en modelos indican una reducción en la precipitación media anual y un aumento en la temperatura del aire de la superficie hacia finales del siglo XXI para la zona centro sur de Chile (Garreaud et al., 2017; Stehr, Debels, Arumi, Alcayaga, \& Romero, 2010). En estudios realizados en la cuenca del río Bío Bío ( $37^{\circ} \mathrm{S}$ a $38^{\circ} \mathrm{S}$ ), Barría et al. (2017) prevén reducciones de escorrentía en zonas con clima mediterráneo y, en particular, en cuencas de gran elevación de Chile. Por su parte, Stehr et al. (2010) indicaron que se producirá una reducción en la magnitud de los caudales medios mensuales y a nuales, siendo tal variación mayor en las épocas de primavera y verano. En consecuencia, se prevé que este tipo de escenarios continúe en el futuro (Quintana \& Aceituno, 2012), presentando mayor recurrencia de sequías (Bosier, Rondanelli, Garreaud, \& Muñoz, 2016). Tal escenario podría intensificar problemas de acceso al recurso, pues la principal fuente de abastecimiento hídrico del centro sur de Chile corresponde a cursos de agua superficial, cuya recarga depende de las precipitaciones (Aguayo, Stehr, \& Link, 2016). Una adecuada consideración de los potenciales impactos del cambio climático sobre los recursos hídricos debiera constituir un requisito previo para emprender cualquier proyecto de largo plazo que esté relacionado con el uso de dichos recursos, para así poder adoptar aquellas estrategias de gestión más apropiadas, evitar efectos ambientales adversos, y conflictos entre los distintos usuarios (Stehr et al., 2010). De este modo, una reforma al Código de Aguas chileno debiera instituir una forma de asignación de derechos y su caducidad, estableciendo la gestión integrada de las cuencas hidrológicas y su institucionalidad. La idea es que el Estado cuente con 
mayores herramientas legales para detener la sobreexplotación del agua.

El resultado de este estudio se une a varios artículos y reportes gubernamentales que sugieren un problema generalizado del abastecimiento del recurso desde el norte al centro sur de Chile. Los resultados ponen a la cuenca del río Ñuble al nivel de lo observado en cuencas con clima mediterráneo del centro de Chile, con precipitaciones concentradas en el invierno austral, y con una media anual que oscila entre 100 y 500 mm en el Valle Central (Viale \& Garreaud, 2015). Son los casos de las cuencas de los ríos La Ligua y Petorca ( 32 $\mathrm{S}$ ) como uno de los más emblemáticos (Budds, 2012). Estas cuencas han sido calificadas como críticas debido a su vulnerabilidad ante la variabilidad hidrológica, en especial sequías. Según el estudio de disponibilidad realizado por la DGA (2006), las cuencas tenían los recursos permanentes agotados, y los eventuales sólo disponibles desde abril hasta noviembre. Por otra parte, Bolados et al. (2017) mencionaron que esta situación ya se producía desde la década de 1990 sobre aguas superficiales, y desde 2000 sobre aguas subterráneas, generando consecuencias socioeconómicas y desgaste ambiental. Al inicio de la década de 2000, las cuencas de los ríos Aconcagua ( $32^{\circ} \mathrm{S}$ a $33^{\circ} \mathrm{S}$ ) (DGA, 2004c) y Maipo ( $33^{\circ} \mathrm{S}$ a $\left.34^{\circ} \mathrm{S}\right)$ (DGA, 2003) tampoco contaban con recursos disponibles durante todo el año para constituir nuevos DdA en ciertas secciones. Esto era corroborado en la práctica con el hecho de que, en los años secos, los ríos debían entrar en turno, no pudiendo las asociaciones de canalistas captar la totalidad del derecho que les correspondía. 
Más al sur de los ejemplos anteriores, donde las precipitaciones tienden a ser mayores, la disponibilidad también es mayor. Por ejemplo, se tiene el caso de la cuenca del río Maule ( $35^{\circ} \mathrm{S}$ a $\left.36^{\circ} \mathrm{S}\right)$, que se encuentra sobreotorgado en ciertas secciones, principalmente entre los meses de noviembre y abril tanto para DdA permanentes como eventuales (DGA, 2005). Incluso se observan situaciones similares al sur del área de estudio ( $\left.\sim 38^{\circ} \mathrm{S}\right)$, donde el clima cambia a templado con influencia mediterránea. La precipitación media anual aumenta a 1000 $1500 \mathrm{~mm}$, con mayor frecuencia de episodios de precipitación durante el verano, relativo a las regiones localizadas más al norte (Viale \& Garreaud, 2015). En esta zona se pueden mencionar estudios como el

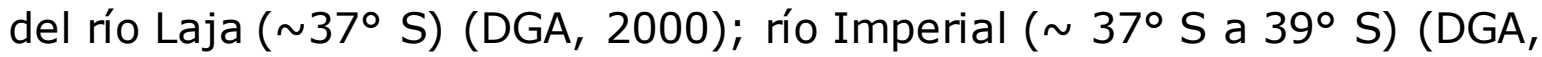
2001), y río Quepe ( $38^{\circ}$ S) (DGA, 2004a), los cuales, en ciertos tramos y sobre todo en verano, poseen sobreotorgamiento de DdA permanentes. En el caso de DdA eventuales, la disponibilidad observada es superior.

De todas formas, es necesario tener en cuenta que estos estudios no son recientes, por lo que una actualización probablemente revelaría mayores sobreotorgamientos. En general, los resultados obtenidos del estudio concuerdan con los ejemplos mostrados y tienen características similares a aquellas cuencas ubicadas entre los límites de las zonas con clima mediterráneo y templado. La disponibilidad de invierno se explica por las mayores precipitaciones de la zona, y el sobreotorgamiento del verano se define por la disminución de precipitaciones frente a la demanda que se mantiene constante. 


\section{Conclusiones}

La evaluación de la disponibilidad hídrica es una herramienta importante para conocer el estado de una cuenca que en un futuro próximo podría desarrollar conflictos debido a la constante disminución de caudales . La generación de políticas públicas enfocadas en una gestión más eficiente del recurso hídrico permitiría evitar tal situación.

Utilizando una metodología que mezcló datos instrumentales y satelitales, se logró identificar sobreotorgamiento de DdA en varios sectores de la cuenca del río Ñuble. Se detectó que el caudal otorgado es mayor al disponible para el ejercicio de DdA consuntivos durante el verano, lo cual condiciona la disponibilidad para otorgar nuevos derechos en la totalidad de la cuenca. En general, el centro de Chile está siendo afectado por estas problemáticas. Su origen se debe tanto a causas climáticas como a la ineficiente gestión; además de la existencia de un marco legal que, guiado por la teoría del libre mercado, no ha podido generar herramientas para la preservación del recurso y garantizar su acceso a la totalidad de la población. La falta de bases de datos confiables, tanto de DdA como de registros hidrológicos instrumentales, son problemas que la administración debiera solucionar 
para ejecutar estudios más confiables. Ese problema con las bases de datos dificulta, por ahora, poder discriminar cuantitativamente entre la sensibilidad de los caudales a los sobreotorgamientos de DdA versus aquella derivada de cambios climáticos regionales que afectan los regímenes pluviométricos.

En cuencas ya sobreotorgadas, una reforma debería proponer medidas que permitan la redistribución de los DdA existentes de la forma más equitativa posible, quitando DdA que no se utilizan de manera productiva, proponiendo medidas que admitan la preservación de la calidad escénica, y desarrollando herramientas para solucionar conflictos de usuarios. Asimismo, los resultados de este trabajo sugieren problemas en el procedimiento administrativo para otorgar DdA. En efecto, es necesario una base de datos actualizada de todos los DdA concedidos, para clarificar las demandas existentes; también se deben mejorar las estimaciones de caudales en estado natural, aumentando la dotación de estaciones pluviométricas y fluviométricas, para contar con registros más confiables.

Así, en el contexto hídrico actual, este problema debería ser abordado de mejor manera por la legislación y, por lo tanto, un cambio del Código de Aguas podría dar soluciones, para abarcar aspectos como la naturaleza de los derechos de agua y el cambio climático.

Por último, el cambio climático, factor que contribuye a la disminución de la oferta hídrica, requiere ser tratado con más atención. Es necesario adaptarse a las nuevas condiciones existentes; por ejemplo, a través de un uso más eficiente del agua, considerándola 
Tecnología y

Ciencias $₫$ Agua
2020, Instituto Mexicano de Tecnología del Agua

Open Access bajo la licencia CC BY-NC-SA 4.0

(https://creativecommons.org/licenses/by-nc-sa/4.0/)

como un bien escaso, implementando tecnologías que permitan la reducción de su consumo.

\section{Referencias}

Aguayo, M., Stehr, A., \& Link, O. (2016). Respuesta hidrológica de una cuenca de meso escala frente a futuros escenarios de expansión forestal. Revista de Geografía Norte Grande, (65), 197-214. DOI: doi.org/10.4067/S0718-34022016000300010

Álvarez-Sevilla, I., Brown-Manrique, O., \& Del Cid-Colindres, E. (2017). Estimación de variables hidrológicas mediante modelos de regresión en la cuenca del río Suchiate, Guatemala. Revista Ciencias Técnicas Agropecuarias, 26(3), 22-29. Recuperado de http://scielo.sld.cu/scielo.php?script=sci_arttext\&pid=S2071 $00542017000300003 \&$ Ing $=$ es\&tIng $=e s$

Barría, P., Peel, M. C., Walsh, K. J., \& Muñoz, A. (2017). The first 300 year streamflow reconstruction of a high-elevation river in Chile using tree rings. International Journal of Climatology, 38(1), 436451. DOI: doi.org/10.1002/joc. 5186

Budds, J. (2012). La demanda, evaluación y asignación del agua en el contexto de escasez: un análisis del ciclo hidrosocial del valle del río La Ligua, Chile. Revista de Geografía Norte Grande, 52, 167184. DOI: doi.org/10.4067/S0718-34022012000200010

Boisier, J. P., Rondanelli, R., Garreaud, R. D., \& Muñoz, F. (2016). Anthropogenic and natural contributions to the Southeast Pacific precipitation decline and recent megadrought in central Chile. 
Tecnología y

Ciencias $₫$ Agua
2020, Instituto Mexicano de Tecnología del Agua

Open Access bajo la licencia CC BY-NC-SA 4.0

(https://creativecommons.org/licenses/by-nc-sa/4.0/)

Geophysical Research Letters, 43(1), 413-421. DOI: doi.org/10.1002/2015GL067265

Bolados, P., Henríquez, F., Ceruti, C., \& Sánchez, A. (2017). La eco-geopolítica del agua: una propuesta desde los territorios en las luchas por la recuperación del agua en la provincia de Petorca (zona central de Chile). Revista Rupturas, 8(1), 159-191. Recuperado de https://investiga.uned.ac.cr/revistas/index.php/rupturas/article/vi ew/1977

Canavos, G. C. (1988). Probabilidad y estadística. Aplicaciones y métodos. México, DF, México: McGraw-Hill/Interamericana de México. Recuperado de https://www.academia.edu/31905164/PROBABILIDAD_Y_ESTAD\% C3\%8DSTICA._Aplicaciones_y_m\%C3\%A9todos._George_C._Can avos.pdf

Carrasco, J. F., Osorio, R., \& Casassa, G. (2008). Secular trend of the equilibrium-line altitude on the western side of the southern Andes, derived from radiosonde and surface observations. Journal of Glaciology, 54(186), 538-550. DOI: doi.org/10.3189/002214308785837002

Costa, E. (2016). Diagnóstico para un cambio: los dilemas de la regulación de las aguas en Chile. Revista Chilena de Derecho, 43(1), 335-354. Recuperado de https://scielo.conicyt.cl/scielo.php?pid=S071834372016000100014\&script=sci_arttext\&tlng=en 
CR2, Centro de Ciencias del Clima y la Resiliencia. (2015). Informe a la Nación. La megasequía 2010-2015: una lección para el futuro. Recuperado de http://repositorio.uchile.cl/bitstream/handle/2250/136717/Megase quia-2010-2015.pdf?sequence $=1$

CR2, Centro de Ciencias del Clima y la Resiliencia. (2017). Explorador climático $(C R)^{2}$. Recuperado de http://explorador.cr2.cl/

DGA, Dirección General de Aguas. (2000). Estudio de disponibilidad cuenca del río Laja (S.I.T. No 70). VIII Región, Chile. Santiago, Chile: Unidad Técnica de la Dirección General de Aguas.

DGA, Dirección General de Aguas. (2001). Plan director para la gestión de los recursos hídricos en la cuenca del río Imperial (S.I.T. No 74). Santiago, Chile: Ayala, Cabrera y Asociados Ltda. Ingenieros Consultores. Recuperado de http://documentos.dga.cl/ADM4187_v1.pdf

DGA, Dirección General de Aguas. (2003). Evaluación de los recursos hídricos superficiales en la cuenca del río Maipo (S.D.T. No 145). Santiago, Chile: Departamento de Administración de Recursos Hídricos.

Recuperado

de http://documentos.dga.cl/ADM2976texto.pdf

DGA, Dirección General de Aguas. (2004a). Análisis de disponibilidad recursos hídricos superficiales cuenca río Quepe (S.D.T. No 175). Santiago, Chile: Departamento de Administración de Recursos. Recuperado de http://documentos.dga.cl/SUP4215.pdf 
DGA, Dirección General de Aguas. (2004b). Diagnóstico y clasificación de los cursos y cuerpos de agua según objetivos de calidad. Cuenca del río Itata. Santiago, Chile: Recuperado de http://portal.mma.gob.cl/wp-content/uploads/2017/12/Itata.pdf

DGA, Dirección General de Aguas. (2004c). Evaluación de los recursos hídricos superficiales en la cuenca del río Aconcagua (S.D.T. No 165). Santiago, Chile: Departamento de Administración de Recursos Hídricos. Recuperado de http://documentos.dga.cl/SUP3099.pdf

DGA, Dirección General de Aguas. (2005). Evaluación de los recursos hídricos superficiales de la cuenca del río Maule (S.D.T. No 197). Santiago, Chile: Departamento de Administración de Recursos Hídricos. Recuperado de http://documentos.dga.cl/SUP4377.pdf

DGA, Dirección General de Aguas. (2006). Evaluación de los recursos hídricos superficiales de las cuencas de los ríos Petorca y La Ligua Va Región (S.D.T. No 226). Santiago, Chile: Departamento de Administración de Recursos Hídricos. Recuperado de http://documentos.dga.cl/SUP4496.pdf

DGA, Dirección General de Aguas. (2008). Manual de normas y procedimientos para la administración de recursos hídricos (S.I.T. No 156). Santiago, Chile: Departamento de Administración de Recursos Hídricos. Recuperado de http://documentos.dga.cl/ADM5016.pdf 
DGA, Dirección General de Aguas. (2017a). Banco Nacional de Aguas. Santiago, Chile. Recuperado de http://snia.dga.cl/BNAConsultas/reportes

DGA, Dirección General de Aguas. (2017b). Derechos de agua. Santiago, Chile. Recuperado de http://derechosdeagua.dga.cl/index.php

Duitama, F. D., Moreno, L. M., \& Zamudio, E. (2015). Estimación de caudales en la cuenca media del río Magdalena empleando el método de transposición de caudales. Revista Tekhne, 12(1), 3948. Recuperado de https://revistas.udistrital.edu.co/index.php/tekhne/article/view/10 440

ESRI, Environmental Systems Research Institute. (2011). Arc Hydro Tools Overview. Esri Water Resources Team. Recuperado de http://downloads.esri.com/blogs/hydro/ah2/arc_hydro_tools_2_0_ overview.pdf

Fernández, A., Muñoz, A., González-Reyes, Á., Aguilera-Betti, I., Toledo, I., Puchi, P., Sauchyn, D., Crespo, S., Frene, C., Mundo, I., González, M., \& Vignola, R. (2018). Dendrohydrology and water resources management in south-central Chile: lessons from the Río Imperial streamflow reconstruction. Hydrology and Earth System Sciences, 22(5), 2921-2935. DOI: doi.org/10.5194/hess22-2921-2018

Garreaud, R. D., Alvarez-Garreton, C., Barichivich, J., Boisier, J. P., Christie, D., Galleguillos, M., \& Zambrano-Bigiarini, M. (2017). The 
2020, Instituto Mexicano de Tecnología del Agua

Open Access bajo la licencia CC BY-NC-SA 4.0

(https://creativecommons.org/licenses/by-nc-sa/4.0/)

2010-2015 megadrought in central Chile: Impacts on regional hydroclimate and vegetation. Hydrology and Earth System Sciences, 21(12), 6307-6327. DOI: doi.org/10.5194/hess-216307-2017

González, P. (2017). Proyecto de Ley que Reforma el Código de Aguas: Tramitación y Principales Modificaciones. Asesoría técnica parlamentaria. Biblioteca del Congreso Nacional de Chile/ BCN. Departamento de Estudios, Extensión y Publicaciones. Recuperado de

https://www.bcn.cl/obtienearchivo?id=repositorio/10221/24620/2 /PL_Reforma_el_C\%C3\%B3digo_de_Aguas.pdf

Larraín, S. (2006). El agua en Chile: entre los derechos humanos y las reglas del mercado. Polis. Revista Latinoamericana, (14). Recuperado de http://journals.openedition.org/polis/5091

Ministerio de Justicia. (2018). Decreto con Fuerza de Ley. Código de Aguas de $1981 . \quad$ Recuperado de https://www.leychile.cl/Navegar?idNorma=5605

Ministerio del Medio Ambiente. (2015). Decreto 71. Reglamento para la determinación del caudal ecológico mínimo. Recuperado de https://www.leychile.cl/Navegar?idNorma $=1073494 \& i d V e r s i o n=20$ 15-01-15

MINVU, Ministerio de Vivienda y Urbanismo. (2002). Observatorio Urbano. Santiago, Chile. Recuperado de http://observatoriourbano.minvu.cl/indurb/wp_indicadores.asp 
Tecnología y

Ciencias $₫$ Agua
2020, Instituto Mexicano de Tecnología del Agua

Open Access bajo la licencia CCBY-NC-SA 4.0

(https://creativecommons.org/licenses/by-nc-sa/4.0/)

Muñoz, A. A., González-Reyes, A., Lara, A., Sauchyn, D., Christie, D., Puchi, P., Urrutia-Jalabert, R., Toledo-Guerrero, I., Aguilera-Betti, I., Mundo, I., Sheppard, P. R., Stahle, D., Villalba, R., Szejner, P., LeQuesne, C., \& Vanstone, J. (2016). Streamflow variability in the Chilean Temperate-Mediterranean climate transition ( $35^{\circ} \mathrm{S}-42^{\circ} \mathrm{S}$ ) during the last 400 years inferred from tree-ring records. Climate Dynamics, 47(12), 4051-4066. DOI: doi.org/10.1007/s00382016-3068-9

NASA, National Aeronautics and Space Administration. (2015). Mirador. Recuperado de https://mirador.gsfc.nasa.gov/collections/TRMM_3B43_007.shtml

NASA, National Aeronautics and Space Administration. (2017). Giovanni. Recuperado de https://giovanni.gsfc.nasa.gov/giovanni/

NOAA, National Oceanic and Atmospheric Administration. (2018). Climate Prediction Center. Recuperado de http://origin.cpc.ncep.noaa.gov/products/analysis_monitoring/ens ostuff/ONI_v5.php

Quintana, J. M., \& Aceituno, P. (2012). Changes in the rainfall regime along the extratropical west coast of South America (Chile): 30 $43^{\circ}$ S. Atmósfera, 25(1), 1-22. Recuperado de www.scielo.org.mx/pdf/atm/v25n1/v25n1a1.pdf

Stehr, A., \& Aguayo, M. (2017) Snow cover dynamics in Andean watersheds of Chile (32.0-39.5 S) during the years 2000-2016. Hydrology and Earth System Sciences, 21(10), 5111-5126. doi.org/10.5194/hess-21-5111-2017 
Stehr, A., Debels, P., Arumi, J. L., Alcayaga, H., \& Romero, F. (2010). Modelación de la respuesta hidrológica al cambio climático: experiencias de dos cuencas de la zona centro-sur de Chile. Tecnología y ciencias del agua, 1(4), 37-58. Recuperado de http://www.scielo.org.mx/scielo.php?script=sci_arttext\&pid=S200 7-24222010000400002

Ugarte, P. (2003). Derecho de aprovechamiento de aguas. Análisis histórico, extensión y alcance en la legislación vigente (Memoria de grado). Santiago, Chile: Universidad de Chile, Departamento de Derecho Económico. Recuperado de http://repositorio.uchile.cl/bitstream/handle/2250/115224/deugarte_p.pdf?sequence $=1$

Universidad de Chile. (s.f.). Universidad de Chile. Santiago, Chile. Recuperado de http://www.uchile.cl/portal/presentacion/la-u-ychile/acerca-de-chile/8069/relieves-de-chile

Urrutia-Jalabert, R., González, M. E., González-Reyes, A., Lara, A., \& Garreaud, R. (2018). Climate variability and forest fires in central and South-Central Chile. Ecosphere, 9(4), 1-17. DOI: doi.org/10.1002/ecs2.2171

USGS, US Geological Survey. (2014). EarthExplorer. Recuperado de https://earthexplorer.usgs.gov/

Valdés-Pineda, R., Pizarro, R., García-Chevesich, P., Valdés, J. B., Olivares, C., Vera, M., Balocchi, F., Pérez, F., Vallejos, C., Fuentes, R., Abarza, A., \& Helwig, B. (2014). Water Governance in Chile: Availability, Management and Climate Change. Journal of 
Tecnología y

Ciencias $₫$ Agua
2020, Instituto Mexicano de Tecnología del Agua

Open Access bajo la licencia CC BY-NC-SA 4.0

(https://creativecommons.org/licenses/by-nc-sa/4.0/)

Hydrology 519, 2538-2567. Recuperado de https://doi.org/10.1016/j.jhydrol.2014.04.016

Vergara, A. (2015). Modelo y mercado de derechos de aguas en Chile: elementos configuradores. En: Urteaga, P., \& Verona, A. (eds.). Cinco años de la ley de recursos hídricos en el Perú. Segundas Jornadas de Derecho de Aguas (pp. 293-306). Recuperado de http://vergarablanco.cl/2015-modelo-mercado-derecho-aguaschile-elementos-configuradores/

Viale, M., \& Garreaud, R. (2015). Orographic effects of the subtropical and extratropical Andes on upwind precipitating clouds. Journal of Geophysical Research: Atmospheres, 120(10), 4962-4974. DOI: doi.org/10.1002/2014JD023014 